\title{
MINIMAL EXCESSIVE MEASURES AND FUNCTIONS ${ }^{1}$
}

\author{
BY
}

E. B. DYNKIN

\begin{abstract}
Let $\boldsymbol{H}$ be a class of measures or functions. An element $\boldsymbol{h}$ of $\boldsymbol{H}$ is minimal if the relation $h=h_{1}+h_{2}, h_{1}, h_{2} \in H$ implies that $h_{1}, h_{2}$ are proportional to $h$. We give a limit procedure for computing minimal excessive measures for an arbitrary Markov semigroup $T_{t}$ in a standard Borel space $E$. Analogous results for excessive functions are obtained assuming that an excessive measure $\gamma$ on $E$ exists such that $T_{t} f=0$ if $f=0 \gamma$-a.e. In the Appendix, we prove that each excessive element can be decomposed into minimal elements and that such a decomposition is unique.
\end{abstract}

\section{Introduction.}

1.1. In 1941 R. S. Martin [13] published a paper where positive harmonic functions in a domain $D$ of a Euclidean space were investigated. Let $H$ stand for the class of all such functions subject to condition $f(a)<\infty$ where $a$ is a fixed point of $D$. Martin has proved that:

(a) each element of $H$ can be decomposed in a unique way into minimal elements normalized by the condition $f(a)=1$;

(b) if the Green function of the Laplacian in $D$ is known, then all minimal elements can be computed by a certain limit process.

J. L. Doob [2] has discovered that the Martin decomposition of harmonic functions is closely related to the behaviour of Brownian paths at the first exit time from $D$. G. A. Hunt [9] has shown that, using these relations, it is possible to get Martin's results by probabilistic considerations. Actually only discrete Markov chains were treated in [1] and [5], however, the methods are applicable to Brownian motion as well.

In [10] Hunt has studied Markov processes with a continuous time parameter on a separable locally compact space and he has proved results of Martin type under certain regularity conditions for the transition functions. The regularity conditions were relaxed by $H$. Kunita and T. Watanabe [11] and by the author [3], [4]. Now we are able to eliminate them completely and to develop a theory applicable to arbitrary Markov processes in standard Borel spaces. In particular, the theory is easy to apply to general diffusion processes without any restrictions on diffusion

Received by the editors March 14, 1979 and, in revised form, April 30, 1979. Presented in an invited address at the annual meeting of the Institute of Mathematical Statistics, August 13-16, 1979 in Washington, D. C.

AMS (MOS) subject classifications (1970). Primary 60J50; Secondary 60J45, 28 A65.

Key words and phrases. Martin boundary, excessive measures and functions, entrance and exit laws, decomposition into minimal (extreme) elements.

${ }^{1}$ Research supported by NSF Grant No. MCS 77-03543. 
and drift coefficients. (In the case of Brownian motion we get in this way a new proof of Martin's results.)

The decomposition of excessive measures and functions into minimal elements was studied in [6] and [7]. In the present paper we concentrate on computation of minimal elements. In the Appendix, we give a new proof of existence and uniqueness of the decomposition into minimal elements. This proof is based on constructing sufficient statistics for certain classes of Markov processes.

1.2. We say that a function $f$ is positive if it takes values from the extended real half-line $[0,+\infty]$. We write $f \in \mathscr{B}$ if $f$ is a positive function measurable with respect to a $\sigma$-algebra $\mathscr{B}$. If $m$ is a measure on $\mathscr{B}$, we write $f \in L^{1}(m)$ if $f$ is $\mathscr{B}$-measurable and $m$-integrable, and we write $f \in L_{+}^{1}(m)$ if in addition $f>0$ $m$-a.e. We denote by $m(f)$ the integral of $f$ with respect to $m$.

\section{Discussion of results.}

2.1. Let $(E, \mathscr{B})$ be a measurable space. A function $p_{t}(x, B), t>0, x \in E$, $B \in \mathscr{B}$ is called a stationary transition function if it is $\mathscr{B}$-measurable in $x$, is a measure with respect to $B$ and if

$$
\begin{gathered}
p_{t}(x, E) \leqslant 1 \text { for all } s, x ; \\
\int_{E} p_{s}(x, d y) p_{t}(y, B)=p_{s+t}(x, B) \text { for all } 0<s<t, x \in E, B \in \mathscr{B} .
\end{gathered}
$$

If (2.2) but not necessarily (2.1) holds, we call $p$ a generalized stationary transition function.

We put

$$
m T_{t}(B)=\int_{E} m(d x) p_{t}(x, B)
$$

and

$$
T_{t} h(x)=\int_{E} p_{t}(x, d y) h(y)
$$

These formulae are meaningful for all measures $m$ on $\mathscr{B}$ and all functions $h \in \mathscr{B}$. We say that $m$ is an excessive measure if it is $\sigma$-finite and if, for each $B \in \mathscr{B}$,

$$
m T_{t}(B) \uparrow m(B) \text { as } t \downarrow 0 .
$$

A function $h \in \mathscr{B}$ is called excessive ${ }^{2}$ if it is finite a.s. with respect to all measures $p_{t}(x, \cdot)$ and if, for every $x \in E$,

$$
T_{t} h(x) \uparrow h(x) \text { as } t \downarrow 0 .
$$

2.2. Throughout this paper we assume that:

2.2.A. $(E, \mathscr{B})$ is a standard Borel space.

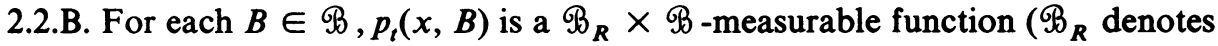
the $\sigma$-algebra of all Borel subsets of the real line $R$ ).

\footnotetext{
${ }^{2}$ Hunt's definition of excessive functions requires measurability with respect to the completion of $\mathscr{B}$ relative to an arbitrary probability measure. This looks less restrictive than $\mathscr{B}$-measurability. However, under the assumption 2.2.B, both conditions are equivalent for functions $h$ with the property (2.5.a).
} 
In all propositions on excessive functions we assume in addition that:

2.2.C. All the measures $p_{t}(x, \cdot)$ are absolutely continuous with respect to a $\sigma$-finite measure $\gamma$.

The role of this condition is revealed by the following lemma.

LEMMA 2.1. Under condition 2.2.C, a Radon-Nikodým derivative

$$
\rho_{t}(x, y)=p_{t}(x, d y) / \gamma(d y)
$$

can be chosen to be measurable in $x, y$ and to satisfy the relation

$$
\int_{E} \rho_{s}(x, y) \gamma(d y) \rho_{t}(y, z)=\rho_{s+t}(x, z)
$$

for all $x, z \in E, s, t>0$. If the measure $\gamma$ is excessive, then we can assume in addition, that

$$
\int \gamma(d x) \rho_{t}(x, y)<1
$$

for all $t, y$.

We say that $\gamma$ is a reference measure if $\gamma(B)=0$ if and only if $p_{t}(x, B)=0$ for all $t$ and $x$. If a measure $\gamma$ satisfies condition 2.2.C, then

$$
\gamma_{1}(B)=\int_{0}^{\infty} d t e^{-t} \int_{E} \gamma(d x) p_{t}(x, B)
$$

is a reference measure. Obviously all reference measures are equivalent, and each excessive measure $\gamma$ satisfying 2.2.C is a reference measure.

2.3. We fix a stationary transition function $p$ and we denote by $M$ the set of all excessive measures and by $H$ the set of all excessive functions.

All minimal elements of $M$ can be obtained by passage to a limit from the Green measure $g_{x}, x \in E$, and the truncated Green measure $g_{x}^{u}, x \in E, u>0$, which are defined by the following formulae

$$
\begin{aligned}
& g_{x}(B)=\int_{0}^{\infty} p_{t}(x, B) d t \\
& g_{x}^{u}(B)=\int_{0}^{u} p_{t}(x, B) d t
\end{aligned}
$$

We say that an element $m$ of $M$ is conservative and we write $m \in M_{c}$ if $g_{x}(l)=\infty$ a.s. $m$ for all strictly positive measurable $l$. We say that $m \in M$ is dissipative and we write $m \in M_{d}$ if $g_{x}(l)<\infty$ a.s. $m$ for all $m$-integrable positive $l$.

LEMMA 2.2. Each minimal element $m$ of $M$ belongs either to $M_{c}$ or to $M_{d}$. If $m \in M_{c}$ then $m T_{t}=m$ for all $t>0$.

TheOReM 2.1. Let a minimal element $m$ of $M$ belong to $M_{c}$. If $\varphi, \psi \in L^{1}(m)$, $m(\psi) \neq 0$, then

$$
m(\varphi) / m(\psi)=\lim _{u \rightarrow \infty}\left(g_{x}^{u}(\varphi) / g_{x}^{u}(\psi)\right)
$$

for $m$-almost all $x$.

Theorem 2.1 is true for all generalized stationary transition functions. 
TheOREM 2.2. Let a minimal element $m$ of $M$ belong to $M_{d}$. There exists a probability measure $P$ on the space $E^{\infty}$ of all sequences $x_{1}, x_{2}, \ldots, x_{k}, \ldots \in E$ such that, if $\varphi, \psi \in L^{1}(m), m(\psi) \neq 0$, then

$$
m(\varphi) / m(\psi)=\lim \left(g_{x_{k}}(\varphi) / g_{x_{k}}(\psi)\right)
$$

for $P$-almost all sequences $\left\{x_{k}\right\}$.

2.4. The following implications of Theorems 2.1-2.2 rather than the theorems themselves are useful for practical computation of minimal elements.

Corollary. Let $S$ be a countable family of positive $\mathscr{B}$-measurable functions. We write $m=S$ - $\lim m_{k}$ if $m_{k}(\varphi) \rightarrow m(\varphi)$ for all $m$-integrable functions $\varphi \in S$. Let $m$ be a minimal element of $M$. If $m$ is conservative, then

$$
m=S-\lim _{u \rightarrow \infty} c(u) g_{x}^{u} \text { for some } x \in E .
$$

If $m$ is dissipative, then

$$
m=S-\lim c_{k} g_{x_{k}} \text { for some } x_{1}, x_{2}, \ldots \in E .
$$

Here $c$ are constants (which can be expressed by formulae

$$
c(u)=m(\psi) / g_{x}^{u}(\psi), \quad c_{k}=m(\psi) / g_{x_{k}}(\psi)
$$

for an arbitrary function $\left.\psi \in L_{+}^{1}(m)\right)$.

2.5. Now let $\gamma$ be a reference measure and let

$$
\begin{aligned}
g^{y}(x) & =\int_{0}^{\infty} \rho_{t}(x, y) d t, \\
g_{u}^{y}(x) & =\int_{0}^{u} \rho_{t}(x, y) d t
\end{aligned}
$$

where $\rho$ is described in Lemma 2.1. We call $g^{y}$ the Green function and $g_{u}^{y}$ the truncated Green function.

Put $\gamma^{h}(d x)=h(x) \gamma(d x), \gamma^{y}(d x)=g^{y}(x) \gamma(d x)$. An element $h$ of $H$ is called conservative if $\gamma^{y}(\varphi)=\infty \gamma^{h}$-a.e. for each strictly positive $\varphi$, and it is called dissipative, if $\gamma^{y}(\varphi)<\infty \gamma^{h}$-a.e. for each $\gamma^{h}$-integrable positive $\varphi$. These definitions are independent of the choice of reference measure. The set of all conservative elements of $H$ is denoted by $H_{c}$ and the set of all dissipative elements by $H_{d}$.

LEMMA 2.3. Suppose that condition 2.2.C holds for an excessive measure $\gamma$. Then each minimal element $h$ of $H$ belongs either to $H_{c}$ or to $H_{d}$. If $h \in H_{c}$ then $T_{t} h=h$ for all $t>0$.

TheOREM 2.3. Suppose that $h$ is a conservative minimal element of $H$. If $h$ is integrable relative to measures $\xi$ and $\eta$ and if $\eta(h) \neq 0$, then

$$
\xi(h) / \eta(h)=\lim _{u \rightarrow \infty}\left(\xi\left(g_{u}^{y}\right) / \eta\left(g_{u}^{y}\right)\right)
$$

for $\gamma^{h}$-almost all $y$. 
THEOREM 2.4. Let $h$ be a dissipative minimal element of $H$. Then there exists $a$ probability measure $P$ on the space $E^{\infty}$ such that, if $h$ is integrable with respect to $\xi$ and $\eta$ and if $\eta(h) \neq 0$, then

$$
\xi(h) / \eta(h)=\lim \left(\xi\left(g^{y_{k}}\right) / \eta\left(g^{y_{k}}\right)\right)
$$

for almost all sequences $\left\{y_{k}\right\}$.

Corollary. Let $S$ be a countable family of measures on $(E, \mathscr{B})$. We write $f=S-\lim f_{k}$ if $\xi\left(f_{k}\right) \rightarrow \xi(f)$ for all $\xi \in S$ such that $f$ is $\xi$-integrable. Let $h$ be $a$ minimal element of $H$. If $h \in H_{c}$, then

$$
h=S-\lim c(u) g_{u}^{y} \quad \text { for some } y \in E .
$$

If $h \in H_{d}$, then

$$
h=S-\lim c_{k} g^{y_{k}} \quad \text { for some } y_{1}, y_{2}, \ldots \in E
$$

(Constants $c$ can be calculated by the formulae

$$
c(u)=\eta(h) / \eta\left(g_{u}^{y}\right), \quad c_{k}=\eta(h) / \eta\left(g^{y_{k}}\right)
$$

where $\eta$ is an arbitrary measure such that $\eta(h)<\infty$.)

REMARK. For $\xi(B)=1_{B}(x)$, formulae (2.16) and (2.17) can be rewritten in the following form

$$
\begin{gathered}
h(x)=\lim _{u \rightarrow \infty} c(u) g_{u}^{y}(x) \\
h(x)=\lim c_{k} g^{y_{k}}(x)
\end{gathered}
$$

where $c$ are given by formulae (2.18). Let $\nu$ be a $\sigma$-finite measure such that $\nu\{h=\infty\}=0$. By Fubini's theorem, for $\gamma^{h}$-almost all $y$, formula (2.16a) is true for $\nu$-almost all $x$. Analogously, for $P$-almost all sequences $y_{k}$, formula (2.17a) holds for $\nu$-almost all $x$.

2.6. Theorem 2.4 implies immediately Martin's results on minimal positive harmonic functions. The condition 2.2.C is satisfied for Lebesgue measure $\gamma$. The density $\rho_{t}(x, y)$ is symmetric and

$$
\int_{D} g^{y}(x) \gamma(d x)=\int_{D} \int_{0}^{\infty} \gamma(d x) \rho_{t}(x, y) d t=\int_{0}^{\infty} \rho_{t}(y, D) d t=E_{y} \beta
$$

where $\beta$ is the first exit time of Brownian motion from the domain $D$. For a bounded domain $D$, the right side is finite and therefore all elements of $H$ are dissipative. Suppose that $h$ is a minimal element and that $h(a)=1$. According to the remark at the end of Subsection 2.5, there exists a sequence $y_{k} \in E$ such that

$$
h(x)=\lim \left(g^{y_{k}}(x) / g^{y_{k}}(a)\right) \text { for } \gamma \text {-almost all } x \in D \text {. }
$$

Take a convergent subsequence and, changing notations, denote it $y_{k}$ again. If $\lim y_{k}=y \in D$, then $h(x)=g^{y}(x) / g^{y}(a) \gamma$-a.e., hence everywhere in $D$ (because both functions are superharmonic). If $y \in \bar{E}$, then the limit in the right side of (2.19) is a harmonic function ( $g^{y}$ is a harmonic in $D \backslash\{y\}$ and Harnack's inequality implies uniform convergence on each compact subset of $D$ ). The sequence $y_{k}$ 
corresponds to a point of the Martin boundary and the function defined by (2.19) is the minimal harmonic function associated with this point. ${ }^{3}$

We see how small the part of this picture which depends on the analytic properties of classical harmonic functions is.

2.7. Although an explicit description of the measure $P$ in Theorems 2.2 and 2.4 is not important for computing minimal elements, it is instructive from the point of view of stochastic processes.

Consider a decreasing sequence $t_{n}, n=0,1, \ldots$, and a sequence of $\sigma$-finite measures $\nu_{t_{n}}$ subject to the condition

$$
\nu_{t_{n}} T_{t_{n-1}-t_{n}}=\nu_{t_{n-1}}, \quad n=1,2, \ldots
$$

Let $l$ be a positive function and $\nu_{t_{0}}(l)=1$. Formulae

$$
\begin{gathered}
m_{t_{0}}\left(d x_{0}\right)=\nu_{t_{0}}\left(d x_{0}\right) l\left(x_{0}\right), \\
m_{t_{n} t_{n-1} \ldots t_{0}}\left(d x_{n}, d x_{n-1}, \ldots, d x_{0}\right) \\
=\nu_{t_{n}}\left(d x_{n}\right) p_{t_{n-1}-t_{n}}\left(x_{n}, x_{n-1}\right) \cdots p_{t_{0}-t_{1}}\left(x_{1}, d x_{0}\right) l\left(x_{0}\right)
\end{gathered}
$$

define a compatible family of finite-dimensional distributions, and by Kolmogorov's theorem, there exists a sequence $X_{t_{n}}$ of random variables taking values in $E$ such that $m_{t_{n} t_{n-1}} \ldots t_{0}$ is the probability distribution of $X_{t_{n}}, X_{t_{n-1}}, \ldots, X_{t_{0}}$. In other words, there exists a measure $P$ in $E^{\infty}$ such that $m_{t_{n}} \cdots t_{0}\left(\Gamma_{n} \times \cdots \times \Gamma_{0}\right)$ is the measure of the cylinder with the base $\Gamma_{n} \times \cdots \times \Gamma_{0}$.

Now each minimal element $m$ of $M$ is either invariant, i.e., $m T_{t}=m$ for all $t$, or $m T_{t} \downarrow 0$ as $t \rightarrow \infty$ (in the second case we call $m$ null-excessive).

If $m$ is invariant, then (2.20) is satisfied for $\nu_{t_{0}}=\nu_{t_{1}}=\cdots=\nu_{t_{n}}=\cdots=m$. For $m \in M_{d}$, Theorem 2.2 holds for every measure $P$ corresponding to a sequence $\nu_{t_{n}}=m, t_{n} \downarrow-\infty$.

If $m$ is null-excessive, then it can be represented in the form

$$
m=\int_{0}^{\infty} \nu_{t} d t
$$

where

$$
\nu_{s} T_{t-s}=\nu_{t} \text { for all } 0<s<t .
$$

Theorem 2.2 holds for every measure $P$ corresponding to a sequence $\nu_{t_{n}}, t_{n} \downarrow 0$.

The random variables $X_{t_{n}}$ form a Markov process. It is natural to interpret the set of minimal elements of $M$ (with proportional elements identified) as the entrance space for this process. This space consists of two parts: the entrance space at time 0 corresponding to the null-excessive elements and the entrance space at $-\infty$ corresponding to the invariant elements.

\footnotetext{
${ }^{3}$ The points corresponding to the minimal harmonic functions form only a part of the Martin boundary. The fundamental results on minimal positive harmonic functions described in Subsection 1.1 have been obtained originally by using a representation of harmonic functions as integrals over all the boundary. The subsequent development has shown that not the entire boundary but only its minimal part is of real importance.
} 
Using (2.21) for a fixed $t_{0}$ and variable $t_{1}, t_{2}, \ldots$, it is possible to define a Markov process $X_{t}$ for all $t \leqslant t_{0}$. The statement of Theorem 2.2 remains true if $t$ tends to 0 or $-\infty$ over any countable subset of $\left(-\infty, t_{0}\right)$. It can take all real values if the paths of $X_{t}$ have certain regularity properties (as in the case of Brownian motion).

2.8. To construct a measure $P$ mentioned in Theorem 2.4 , we consider an increasing sequence $t_{n}, n=0,1, \ldots$, a sequence of positive measurble functions $\varphi^{t_{n}}$ subject to conditions

$$
T_{t_{n}-t_{n-1}} \varphi^{t_{n}}=\varphi^{t_{n-1}}, \quad n=1,2, \ldots,
$$

and a measure $\nu$ such that $\nu\left(\varphi^{t_{0}}\right)=1$. Let $P$ be a measure on $E^{\infty}$ corresponding to the finite-dimensional distributions

$$
\begin{gathered}
m_{t_{0}}\left(d x_{0}\right)=\nu\left(d x_{0}\right) \varphi^{t_{0}}\left(x_{0}\right) \\
m_{t_{0} t_{1}} \ldots t_{n}\left(d x_{0}, d x_{1}, \ldots, d x_{n}\right) \\
=\nu\left(d x_{0}\right) p_{t_{1}-t_{0}}\left(x_{0}, d x_{1}\right) \cdots p_{t_{n}-t_{n-1}}\left(x_{n-1}, d x_{n}\right) \varphi^{t_{n}}\left(x_{n}\right) .
\end{gathered}
$$

If a minimal element $h$ of $H$ is invariant (i.e., $T_{t} h=h$ for all $t$ ), then Theorem 2.4 holds for a measure $P$ corresponding to $\varphi^{t_{n}}=h, t_{n} \uparrow+\infty$. If $h$ is null-excessive (i.e., $T_{t} h \downarrow 0$ as $\left.t \rightarrow \infty\right)$, then

$$
h=\int_{-\infty}^{0} \varphi^{t} d t
$$

where

$$
T_{t-s} \varphi^{t}=\varphi^{s} \text { for all } s<t<0,
$$

and we can use any measure $P$ corresponding to $\varphi^{t_{n}}$ and $t_{n} \uparrow 0$.

The set of minimal elements of $H$, with the identification of proportional elements, can be interpreted as the exit space at time $\mathbf{0}$ for null-excessive elements, and at time $+\infty$ for invariant elements. (Time 0 can be replaced with any other finite time $s_{0}$.)

From a probabilistic point of view, it is more natural to consider a stochastic process with random birth time $\alpha$ and death time $\beta$ and to interpret elements of the entrance and exit spaces as possible birth and death places (cf. Theorems 7.2 and 7.4).

\section{Conservative minimal elements.}

3.1. A function $v(x, B), x \in E, B \in \mathscr{B}$, is called a kernel if it is a $\mathscr{B}$-measurable function of $x$ for each $B \in \mathscr{B}$, and is a measure relative to $B$ for each $x \in E$. A kernel $v(x, B)$ defines a transformation of measures

$$
m V(B)=\int_{E} m(d x) v(x, B)
$$

and a transformation of positive measurable functions

$$
V \varphi(x)=\int_{E} v(x, d y) \varphi(y) .
$$


Two kernels $v$ and $v^{*}$ are dual relative to a measure $m$ if

$$
\int \varphi(x) V \psi(x) m(d x)=\int \psi(x) V^{*} \varphi(x) m(d x)
$$

for all $\varphi$ and $\psi$.

To each $V$ there correspond the Green operator

$$
G \varphi(x)=\sum_{k=0}^{\infty} V^{k} \varphi(x)
$$

and the truncated Green operator

$$
G_{n} \varphi(x)=\sum_{k=0}^{n-1} V^{k} \varphi(x) .
$$

The following proposition is one form of the Chacon-Ornstein ergodic theorem.

THEOREM 3.1. Let $v$ be a kernel on $(E, \mathscr{B})$ and let a $\sigma$-finite measure $m$ on $\mathscr{B}$ satisfy the condition

$$
m V(B) \leqslant m(B) \text { for all } B \in \mathscr{B} .
$$

Then $m=m_{c}+m_{d}$ where

3.1.A. The measures $m_{c}$ and $m_{d}$ are singular with respect to each other,

3.1.B. $G \varphi=0$ or $+\infty m_{c}$-a.e. for each $\varphi \in \mathscr{B}$,

3.1.C. $G \varphi<\infty m_{d}$-a.e. for each $\varphi \in L_{+}^{1}(m)$.

These properties define the measures $m_{c}$ and $m_{d}$ uniquely. For each $l \in L_{+}^{1}(m)$, we have $m_{c}(B)=m\left(B \cap E_{c}\right), m_{d}(B)=m\left(B \cap E_{d}\right)$ where $E_{c}=\{x: G l(x)=\infty\}, E_{d}=$ $\{x: G l(x)<\infty\}$.

The measures $m_{c}$ and $m_{d}$ are called the conservative and dissipative parts of $m$ relative to $V$.

The following statments hold.

3.1.D. If $\varphi=0 m_{d}$-a.e., then $V \varphi=0 m_{d}$-a.e. and $m(V \varphi)=m(\varphi)$.

3.1.E. If $V 1<1 m$-a.e., then the equality $\psi=0 m_{c}$-a.e. implies the equality $V \psi=0 m_{c}-a . e$.

3.1.F. Suppose that $m_{d}=0$. Put $B \in \mathscr{B}_{m}^{V}$ if $B \in \mathscr{B}$ and if

$$
\int_{B} \varphi d m=\int_{B} V \varphi d m \text { for all } \varphi \in \Re
$$

The class $\mathscr{B}_{m}^{V}$ is a $\sigma$-algebra in $\mathscr{B}$ and

$$
\lim \left(G_{n} \varphi(x) / G_{n} \psi(x)\right)=m^{\psi}\left(\frac{\varphi}{\psi} \mid \oiint_{m}^{V}\right)
$$

for $m$-almost all $x$ if $\varphi \in L^{1}(m), \psi \in L_{+}^{1}(m)$. $\left(\right.$ Here $m^{\psi}(d x)=\psi(x) m(d x)$.)

All these statements, except 3.1.E, are proved, e.g., in [14] (\$§V.5 and V.6).

Let us prove 3.1.E. If $V 1<1, m$-a.e., then $m\left(V^{*} \varphi\right)=m(\psi V 1)<m(\psi)$ for all $\psi \in \mathscr{B}$, and $V^{*}$ satisfies condition (3.3). Let $G^{*}$ be the corresponding Green operator. Take $l \in L_{+}^{1}(m)$. As we know, $m_{c}$ and $m_{d}$ are the restrictions of $m$ to $E_{c}=\{G l=\infty\}$ and $E_{d}=\{G l<\infty\}$. Because of 3.1.C, there exists a function $\hat{l} \in L^{1}(m)$ such that $\hat{l}=0$ on $E_{c}, \hat{l}>0$ on $E_{d}$ and $m(\hat{l} G l)=m_{d}(\hat{l} G l)<\infty$. We 
have $m\left(l G^{*} \hat{l}\right)<\infty$, hence $m\left\{G^{*} \hat{l}=\infty\right\}=0$. Let $\hat{m}_{c}$ and $\hat{m}_{d}$ be the conservative and dissipative parts of $m$ relative to $V^{*}$. By 3.1.B $\hat{m}_{c}\left\{0<G^{*} \hat{l}<\infty\right\}=0$ and $\hat{m}_{c}\left\{0<G^{*} \hat{l}\right\}=0$. However $G^{*} \hat{l} \geqslant \hat{l}>0$ on $E_{d}$. Hence $\hat{m}_{c}\left(E_{d}\right)=0$ and, for all $B \in \mathscr{B}, m_{d}(B)=m\left(B \cap E_{d}\right)=\hat{m}_{d}\left(B \cap E_{d}\right) \leqslant \hat{m}_{d}(B)$. Because the roles of $V$ and $V^{*}$ are symmetric, we also have $\hat{m}_{d} \leqslant m_{d}$. Hence $\hat{m}_{d}=m_{d}, \hat{m}_{c}=m_{c}$.

Now let $\varphi=0 m_{d}$-a.e., $\psi=0 m_{c}$-a.e. Then, by 3.1.D, $V^{*} \varphi=0, \hat{m}_{d}=m_{d}$-a.e. and $m_{c}(\varphi V \psi)=m\left(\psi V^{*} \varphi\right)=0$. Hence $V \psi=0 m_{c}$-a.e.

REMARK. Using the relation $G_{n+1} \varphi=G_{n} V \varphi+\varphi$, it is easy to prove that the limit (3.4) coincides $m_{c}$-a.e. with the limits

$$
\lim _{n \rightarrow \infty}\left(G_{n+1} \varphi(x) / G_{n} \psi(x)\right)=\lim _{n \rightarrow \infty}\left(G_{n} \varphi(x) / G_{n+1} \psi(x)\right) .
$$

3.2. Now we apply Theorem 3.1 to investigating the class $M$ of all excessive measures associated with a stationary transition function $p_{t}(x, B)$. Let $T_{t}$ be operators defined by formulas (2.3) and (2.4). Consider the Green measure $g_{x}$ and the truncated Green measure $g_{x}^{u}$ introduced by (2.10) and (2.11).

We put

$$
E_{0}=\left\{x: p_{t}(x, B)=0 \text { for all } t, B\right\}
$$

and we notice that, if $l$ is strictly positive, then

$$
g_{x}(l)>0 \text { on } E \backslash E_{0} \text {. }
$$

Indeed, if $g_{x}(l)=0$, then $p_{t}(x, E)=0$ for almost all $t$, and $x \in E_{0}$ because of (2.2).

LEMMA 3.1. Fix a strictly positive function $l \in \mathscr{B}$ and consider, for each measure $m$ on $\mathscr{B}$, its restrictions $m_{c}$ and $m_{d}$ to the sets

$$
E_{c}=\left\{x: g_{x}(l)=\infty\right\}, \quad E_{d}=\left\{x: g_{x}(l)<\infty\right\} .
$$

If $m \in M$ and $m(l)<\infty$, then $m_{c} \in M_{c}$ and $m_{d} \in M_{d}$. Moreover $m_{c}$ is invariant with respect to operators $T_{t}$.

Proof. $1^{\circ}$. Since (3.3) holds for $V=T_{t}$, we have a decomposition $m=m_{c}^{t}+m_{d}^{t}$ where $m_{c}^{t}$ and $m_{d}^{t}$ satisfy conditions 3.1.A, B, C. For each $\varphi \in \mathscr{B}$

$$
g_{x}(\varphi)=G \tilde{\varphi}(x) \quad \text { where } \tilde{\varphi}(x)=g_{x}^{t}(\varphi) .
$$

It follows from 3.1.B and (3.7) that $m_{d}^{t}\left(E_{c}\right)=0, m_{c}^{t}\left(E_{d} \backslash E_{0}\right)=0$. Besides $G T_{t} l(x)=$ $l(x)$ on $E_{0}$ and, since $0<l<\infty m$-a.e., we have $m_{c}^{t}\left(E_{0}\right)=0$ by 3.1.B. Let $B_{c}, B_{d}$ stand for the intersections of $B \in \mathscr{B}$ with $E_{c}$ and $E_{d}$. We have $m_{c}(B)=m\left(B_{c}\right)=$ $m_{c}^{t}\left(B_{c}\right)=m_{c}^{t}(B)$. Thus $m_{c}=m_{c}^{t}$. Analogously $m_{d}=m_{d}^{t}$.

$2^{\circ}$. Let $\varphi_{c}, \varphi_{d}$ be the restrictions of $\varphi \in \mathscr{B}$ to $E_{c}, E_{d}$. By 3.1.D and 3.1.E, we have

$$
\begin{aligned}
& m_{c}\left(T_{t} \varphi\right)=m_{c}\left(T_{t} \varphi_{c}\right)=m\left(T_{t} \varphi_{c}\right)=m\left(\varphi_{c}\right)=m_{c}(\varphi), \\
& m_{d}\left(T_{t} \varphi\right)=m_{d}\left(T_{t} \varphi_{d}\right)=m\left(T_{t} \varphi_{d}\right) \uparrow m\left(\varphi_{d}\right)=m_{d}(\varphi) \text { as } t \downarrow 0 .
\end{aligned}
$$

Hence $m_{c}$ and $m_{d}$ belong to $M$ and $m_{c}$ is invariant with respect to $T_{t}$.

$3^{\circ}$. It follows from 3.1.B, C, (3.6) and (3.7), that $g_{x}(\varphi)=\infty, m_{c}=m_{c}^{t}$-a.e. if $\varphi>0$, and $g_{x}(\varphi)<\infty, m_{d}=m_{d}^{t}$-a.e. if $\varphi>0, m(\varphi)<\infty$. Hence $m_{c} \in M_{c}, m_{d} \in$ $M_{d}$. 
3.3. Evidently Lemma 3.1 implies Lemma 2.2. Theorem 2.1 follows from the following result.

Theorem 3.2. Let $m \in M_{c}$. Put $B \in \mathscr{B}_{m}^{T}$ if $B \in \mathscr{B}$ and

$$
\int_{B} \varphi d m=\int_{B} T_{t} \varphi d m \text { for all } t>0, \varphi \in \mathscr{B} \text {. }
$$

The class $\mathscr{B}_{m}^{T}$ is a $\sigma$-algebra in $E$. If $\varphi \in L^{1}(m), \psi \in L_{+}^{1}(m)$, then

$$
\lim _{u \rightarrow \infty}\left(g_{x}^{u}(\varphi) / g_{x}^{u}(\psi)\right)=m^{\psi}\left(\frac{\varphi}{\psi} \mid \Re_{m}^{T}\right)
$$

for $m$-almost all $x$. If $m$ is a minimal element of $M$, then the right side of (3.9) is equal to $m(\varphi) / m(\psi)$.

Proof. We apply part 3.1.F of Theorem 3.1 to $V=T_{t}$. Notice that $G_{n}$ in formula (3.4) and $g_{x}^{u}$ in (2.11) are connected by the relation

$$
G_{n} \tilde{\varphi}(x)<g_{x}^{u}(\varphi)<G_{n+1} \tilde{\varphi}(x) \text { for } n t<u<(n+1) t
$$

where $\tilde{\varphi}(x)=g_{x}^{t}(\varphi)$. We can write (3.10) in the following form

$$
G_{n} \tilde{\varphi} / c_{x}(u) \leqslant g_{x}^{u}(\varphi) / c_{x}(u) \leqslant G_{n+1} \tilde{\varphi} / c_{x}(u)
$$

for $n t<u<(n+1) t$ where $c_{x}(u)=G_{n} \psi(x)$ for $n t \leqslant u<(n+1) t$. Since $m(\tilde{\varphi})=$ $\operatorname{tm}(\varphi)<\infty$, it follows from (3.4), (3.5) and (3.11) that

$$
\lim _{u \rightarrow \infty}\left(g_{x}^{u}(\varphi) / c_{x}(u)\right)=m^{\psi}\left(\frac{\tilde{\varphi}}{\psi} \mid \mathscr{B}_{m}^{V}\right), \quad m \text {-a.e. }
$$

It is easy to check that the right side of (3.12) equals $\operatorname{tm}^{\psi}\left(\varphi / \psi \mid \mathscr{B}_{m}^{V}\right)$. Since this expression is equal to $t$ for $\varphi=\psi$, we have from (3.12)

$$
\lim _{u \rightarrow \infty}\left(g_{x}^{u}(\varphi) / g_{x}^{u}(\psi)\right)=m^{\psi}\left(\frac{\varphi}{\psi} \mid \Re_{m}^{V}\right), \quad m \text {-a.e. }
$$

Denote by $F$ the left side of (3.13) (on the set where the limit does not exist, we replace it by lim sup). It follows from (3.13) that $F \in \mathscr{B}_{m}^{V}$ for all $V=T_{t}$. Hence $F \in \mathscr{B}_{m}^{T}$ and (3.13) implies (3.9).

It is easy to see that if $m \in M_{c}$, then its restriction $m_{B}$ to any set $B \in \mathscr{B}_{m}^{T}$ belongs to $M$. If $m$ is minimal then $m_{B}$ is proportional to $m$, hence $m(B)=0$ or $m(E \backslash B)=0$. Therefore each $\mathscr{B}_{m}^{T}$-measurable function is constant a.s. $m$. The last statement of Theorem 3.2 follows easily from this observation.

REMARK. In Subsections 3.2, 3.3, only the proof of Lemma 3.1 makes use of 3.1.E and therefore depends on the part (2.1) of the definition of a stationary transition function. The rest is valid for generalized transition functions as well.

3.4. Proof of Lemma 2.1. We start from any version $\bar{\rho}_{t}(x, y)$ of the RadonNikodým derivative $p_{t}(x, d y) / \gamma(d y)$ measurable in $x, y$ and we put

$$
\begin{aligned}
\rho_{s}^{r}(x, z) & =\int \bar{\rho}_{s-r}(x, y) \gamma(d y) \bar{\rho}_{r}(y, z) \\
& =\int p_{s-r}(x, d y) \bar{\rho}_{r}(y, z), \quad 0<r<s .
\end{aligned}
$$


We set $z \in E^{\prime}$ if, for all rational $s>r>0$,

$$
\rho_{s}^{r}(x, z)=\bar{\rho}_{s}(x, z) \text { for } \gamma \text {-almost all } x \text {. }
$$

It is easy to check that

$$
\int_{B_{1}} \int_{B_{2}} \gamma(d x) \rho_{s}^{r}(x, z) \gamma(d z)=\int_{B_{1}} \int_{B_{2}} \gamma(d x) \bar{\rho}_{s}(x, z) \gamma(d z)
$$

for all $B_{1}, B_{2} \in \mathscr{B}$. Hence (3.14) holds for $\gamma$-almost all $z$, and $\gamma\left(E \backslash E^{\prime}\right)=0$.

Now if $z \in E^{\prime}$ and $0<r_{1}<r_{2}<s$ are rational, then

$$
\begin{aligned}
\rho_{s}^{r_{2}}(x, z) & =\int p_{s-r_{2}}\left(x, d y_{1}\right) \bar{\rho}_{r_{2}}\left(y_{1}, z\right)=\int p_{s-r_{2}}\left(x, d y_{1}\right) \rho_{r_{2}}^{r_{1}}\left(y_{1}, z\right) \\
& =\int p_{s-r_{2}}\left(x, d y_{1}\right) p_{r_{2}-r_{1}}\left(y_{1}, d y_{2}\right) \bar{\rho}_{r_{1}}\left(y_{2}, z\right) \\
& =\int p_{s-r_{1}}\left(x, d y_{2}\right) \bar{\rho}_{1}\left(y_{2}, z\right)=\rho_{s}^{r_{1}}(x, z),
\end{aligned}
$$

and we can define $\rho_{s}(x, z)$ for $z \in E^{\prime}$ by the formula $\rho_{s}(x, z)=\rho_{s}^{r}(x, z)$ for any rational $r \in(0, s)$. For $z \bar{\in} E^{\prime}$ we put $\rho_{s}(x, z)=\rho_{s}\left(x, z_{0}\right)$ where $z_{0}$ is a fixed element of $E^{\prime}$. Obviously $\rho$ satisfies (2.6) and (2.7).

If $\gamma$ is excessive, then, for each $B \in \mathscr{B}, s>r>0$

$$
\int \gamma(d x) \int_{B} \rho_{s}^{r}(x, z) \gamma(d z)=\int \gamma(d x) p_{s}(x, B) \leqslant \gamma(B)
$$

hence for $\gamma$-almost all $z$

$$
\int \gamma(d x) \rho_{s}(x, z)<1 \text {. }
$$

Put $z \in E^{\prime \prime}$ if $z \in E^{\prime}$ and (3.15) holds. Since $\gamma\left(E^{\prime} \backslash E^{\prime \prime}\right)=0$, we can replace $E^{\prime}$ with $E^{\prime \prime}$ in the definition of $\rho$, and we get a function which satisfies (2.8) as well as (2.6) and (2.7).

3.5. The investigation of excessive functions associated with a stationary transition function $p$ can be reduced to investigating excessive measures associated with another stationary transition function $\hat{p}$.

Suppose that $\gamma \in M$ is a reference measure for $p$ and let $\rho_{t}(x, y)$ be the function defined in Lemma 2.1. Then the formula

$$
\hat{p}_{t}(x, d y)=\gamma(d y) \rho_{t}(y, x)
$$

defines a stationary transition function. Let $\hat{T}_{t}$ be the operators corresponding to $\hat{p}$. For all $\varphi, \psi \in \Re, t>0$

$$
\gamma\left(\varphi T_{t} \psi\right)=\gamma\left(\psi \hat{T}_{t} \varphi\right)
$$

Hence

$$
\gamma\left(\varphi g_{u}^{x}\right)=\hat{g}_{x}^{u}(\varphi), \quad \gamma\left(\varphi g^{x}\right)=\hat{g}_{x}(\varphi)
$$

where $g_{u}^{x}$ and $g^{x}$ are defined by (2.14), (2.15), and

$$
\begin{aligned}
& \hat{g}_{x}^{u}(B)=\int_{0}^{u} \hat{p}_{t}(x, B) d t=\int_{E} \gamma(d y) \int_{B} g_{u}^{y}(x) \gamma(d x), \\
& \hat{g}_{x}(B)=\hat{g}_{x}^{\infty}(B)
\end{aligned}
$$


are the truncated Green measure and the Green measure for $\hat{p}$. Denote by $\hat{M}$ the class of excessive measures for $\hat{p}$. Put

$$
\gamma^{h}(d y)=h(y) \gamma(d y) .
$$

By (3.17) $\gamma^{h}\left(\hat{T}_{t} \varphi\right)=\gamma\left(\varphi T_{t} h\right)$. Hence if $h \in H$, then $\gamma^{h} \in \hat{M}$. Now suppose that $m \in \hat{M}$. Then $\left(m \hat{T}_{t}\right)(B)=\int_{B} a_{t}(y) \gamma(d y) \uparrow m(B)$ as $t \downarrow 0$ where $a_{t}(y)=$ $\int m(d x) p_{t}(x, y)$. This implies the existence of a function $h \in H$ such that $a_{t}(y) \uparrow h(y) m$-a.e. as $t \downarrow 0$ (see [6, $\S 4$ and 5] for details). Obviously $m=\gamma^{h}$. Hence the mapping $h \rightarrow \gamma^{h}$ defined by (3.20) is a 1-1-splitting of $H$ onto $\hat{M}$. It is easy to see that under this mapping the sets of minimal, conservative and dissipative elements of $H$ correspond to analogous subsets of $\hat{M}$. Hence Lemma 2.3 follows from Lemma 2.2 .

3.6. Now we prove Theorem 2.3. Let $h$ be a conservative minimal element of $H$. Then $\gamma^{h}$ is a conservative minimal element of $\hat{M}$. By Theorem 2.1, if $\varphi, \psi \in L^{1}\left(\gamma^{h}\right)$ and $\gamma^{h}(\psi) \neq 0$, then

$$
\gamma^{h}(\varphi) / \gamma^{h}(\psi)=\lim _{u \rightarrow \infty}\left(\hat{g}_{x}^{u}(\varphi) / \hat{g}_{x}^{u}(\psi)\right), \quad \gamma^{h} \text {-a.e. }
$$

(Since Theorem 2.1 holds for generalized stationary transition funcions, we do not need an assumption that the reference measure $\gamma$ is excessive.)

Let $h$ be integrable with respect to a measure $\xi$. Put $\varphi(y)=\int \xi(d z) \rho_{t}(z, y)$. We have

$$
\begin{gathered}
\gamma^{h}(\varphi)=\xi\left(T_{t} h\right)=\xi(h)<\infty \\
\hat{g}_{x}^{u}(\varphi)=\int_{t}^{t+u} d s \int \xi(d z) \rho_{s}(z, x)=\xi\left(g_{t+u}^{x}\right)-\xi\left(g_{t}^{x}\right) .
\end{gathered}
$$

Here $\xi\left(g_{t}^{x}\right)<\infty \gamma^{h}$-a.e. since

$$
\int \xi\left(g_{t}^{x}\right) \gamma^{h}(d x)=\xi\left(\int_{0}^{t} T_{s} h d s\right)=t \xi(h)<\infty
$$

It follows from (3.21), (3.22), (3.23) and (3.24) that

$$
\xi(h) / \gamma^{h}(\psi)=\lim \xi\left(g_{u}^{x}\right) / c(u), \gamma^{h} \text {-a.e. }
$$

where $c(u)=\hat{g}_{x}^{u-t}(\psi)$. This implies Theorem 2.3.

4. Time-dependent excessive measures and functions.

4.1. The space $M$ of excessive measures associated with a stationary transition function is a subset of a larger space $T M$ of time-dependent excessive measures. Put

$$
\begin{gathered}
p(s, x ; t, B)=p_{t-s}(x, B), \\
T_{t}^{s} \varphi(x)=\int p(s, x ; t, d y) \varphi(y), \\
\left(n T_{t}^{s}\right)(B)=\int n(d x) p(s, x ; t, B) .
\end{gathered}
$$

Suppose that for each $t \in R$ a $\sigma$-finite measure $n_{t}$ on $(E, \mathscr{B})$ is given and let $n_{s} T_{t}^{s} \uparrow n_{t}$ as $s \uparrow t$. Then we say that $n$ is a time-dependent excessive measure and we write $n \in T M$. 
An important example of time-dependent excessive measures are entrance laws. We say that an element $n \neq 0$ of $T M$ is an entrance law at time $s_{0}\left(-\infty<s_{0}<\right.$ $+\infty)$ if $n_{t}=0$ for $t \leqslant s_{0}, n_{s} T_{t}^{s}=n_{t}$ for $s_{0}<s<t$.

4.2. Let a positive measurable function $f^{t}$ on $E$ be given for each $t \in R$ and let $f^{t}$ be finite a.e. with respect to all measures $p(s, x ; t,-)$. We say that $f$ is a time-dependent excessive function and we write $f \in T H$ if $f^{t}<\infty$ a.s. with respect to all measures $p(s, x ; t, \cdot)$ and $T_{t}^{s} f^{t} \uparrow f^{s}$ as $t \downarrow s$. An element $f \neq 0$ of $T H$ is called an exit law at time $u_{0},-\infty<u_{0} \leqslant+\infty$, if $f^{t}=0$ for $t>u_{0}, T_{t}^{s} f^{t}=f^{s}$ for $s<t<u_{0}$. It is easy to see that $f^{s}<\infty$ a.s. $n_{s}$ for every $f \in T H, n \in T M$.

4.3. All these definitions are applicable also to nonstationary transition functions $p(s, x ; t, \cdot)$. (In the nonstationary case, the state space $\left(E_{t}, \mathscr{B}_{t}\right)$ can depend on $t$ and $p(s, x ; t, B)$ is defined for $s<t \in R, x \in E_{s}, B \in \mathscr{B}_{t}$.) To get the definition of such functions, we replace conditions (2.1)-(2.2) by

$$
\begin{gathered}
p(s, x ; t, E)<1 \text { for all } s<t \in R, x \in E, \\
\int_{E} p(s, x ; t, d y) p(t, y ; u, B)=p(s, x ; u, B)
\end{gathered}
$$

for all $s<t<u \in R, x \in E, B \in \mathscr{B}$.

We put $p(s, x ; t, B)=0$ for $s \geqslant t$. Obviously $n_{t}(B)=p(s, x ; t, B)$ is an entrance law at time $s$, and $f^{s}(x)=p(s, x ; t, B)$ is an exit law at time $t$.

4.4. Condition 2.2. B implies that, for each $n \in T M$ and every $\varphi \in \mathscr{B}, n_{t}(\varphi)$ is measurable in $t$. Indeed, for every finite set $\Lambda=\left\{t_{1}<t_{2}<\cdots<t_{n}\right\}$, the function

$$
\begin{aligned}
& F_{\Lambda}(t)=0 \text { for } t<t_{1} \text { and } t>t_{n}, \\
& F_{\Lambda}(t)=n_{t_{k}}\left(T_{t}^{t} \varphi\right) \text { for } t_{k}<t<t_{k+1}
\end{aligned}
$$

is measurable in $t$, and $F_{\Lambda_{k}}(t) \rightarrow n_{t}(\varphi)$ if $\Lambda_{k}$ is an increasing sequence with the union everywhere dense in $R$. The same arguments show that if, for each $t, n_{t}(\varphi)$ is a measurable function of a parameter $\omega$, then it is measurable in $t, \omega$.

4.5. Let $c(t), t \in R$, and $l(x), x \in E$, be positive measurable functions and $\gamma$ be a measure on $E$. Then

$$
n_{t}(B)=\int_{-\infty}^{t} d s c(s) \int_{E} \gamma(d x) p(s, x ; t, B)
$$

is a time-dependent excessive measure, and

$$
f^{s}(x)=\int_{s}^{\infty} d t c(t) \int_{E} p(s, x ; t, d y) l(y)
$$

is a time-dependent excessive function.

4.6. Let $p(s, x ; t, B)$ be a nonstationary transition function. Put $x \in E_{s}^{0}$ if $p(s, x$; $t, B)=0$ for all $t, B$. (For a stationary transition function, $E_{s}^{0}$ does not depend on $s$ and coincides with $E^{0}$ defined in Subsection 3.2.) If $f \in T H$, then $f^{s}(x)=0$ for all $x \in E_{s}^{0}$. We set $f \in T H^{+}$if $f^{s}(x)>0$ outside $E_{s}^{0}$.

For each $q \in \mathrm{TH}^{+}$, the formula

$$
\begin{aligned}
p^{q}(s, x ; t, d y) & =q^{s}(x)^{-1} p(s, x ; t, d y) q^{t}(y) \text { for } 0<q^{s}(x)<\infty \\
& =0 \text { if } q^{s}(x)=0 \text { or } q^{s}(x)=\infty
\end{aligned}
$$


defines a new transition function. A function $f$ is a time-dependent excessive function for $p$ if and only if

$$
\begin{aligned}
f_{q}^{t}(x) & =f^{t}(x) / q^{t}(x) \text { for } 0<q^{t}(x)<\infty, \\
& =0 \text { otherwise }
\end{aligned}
$$

is a time-dependent excessive function for $p^{q}$. Analogously $n$ is a time-dependent excessive measure for $p$ if and only if $n_{t}^{q}(d x)=q^{t}(x) n_{t}(d x)$ is a time-dependent excessive measure for $p^{q}$.

\section{Markov processes.}

5.1. A stochastic process on a random time interval is determined by the following elements:

(i) a measure: $(\Omega, \mathcal{F}, P)$,

(ii) two measurable functions $\alpha(\omega)<\beta(\omega)$ on $\Omega$ with values in the extended real line $[-\infty,+\infty]$,

(iii) for each $t \in R$, a measurable mapping $x_{t}(\omega)$ of the set $\{\omega ; \alpha(\omega)<t<\beta(\omega)\}$ into a measurable space $\left(E_{t}, \mathscr{B}_{t}\right)$.

The moments $\alpha$ and $\beta$ are called the birth time and the death time.

We say that $a$ path $\omega$ is given if a point $\omega(t)$ of $E_{t}$ is fixed for each $t$ of an open interval $I \subset R$, and we say that a process $x_{t}$ is canonical if $\Omega$ coincides with the space of all paths, if $x_{t}(\omega)=\omega(t), t \in I=(\alpha(\omega), \beta(\omega))$, and if $\mathcal{F}$ is the minimal $\sigma$-algebra in $\Omega$ which contains the sets

$$
\{\alpha<t\}, \quad\{\beta>t\}, \quad\left\{\alpha<t, x_{t} \in B, \beta>t\right\}
$$

for all $t \in R, B \in \mathscr{B}$.

THeOREM 5.1. Let $p$ be a transition function on a standard Borel space $(E, \mathscr{B})$ and let $n \in T M, f \in T H$. Then there exists a canonical stochastic process $\left(x_{t}, P_{n}^{f}\right)$ such that

$$
\begin{aligned}
P_{n}^{f}\{\alpha & \left.<t_{1}, x_{t_{1}} \in B_{1}, \ldots, x_{t_{k}} \in B_{k}, t_{k}<\beta\right\} \\
& =\int_{B_{1}} \cdots \int_{B_{k}} n_{t_{1}}\left(d x_{1}\right) p\left(t_{1}, x_{1}, t_{2}, d x_{2}\right) \cdots p\left(t_{k-1}, x_{k-1}, t_{k}, d x_{k}\right) f^{t_{k}}\left(x_{k}\right)
\end{aligned}
$$

for all $k=1,2, \ldots$, all $t_{1}<t_{2}<\cdots<t_{k} \in R$ and $B_{1}, \ldots, B_{k} \in \mathscr{B}$. We have

$$
P_{n}^{f}(\Omega)=\langle n, f\rangle
$$

where $\langle n, f\rangle$ is the supremum, over all finite sets $\Lambda=\left\{t_{1}<\cdots<t_{k}\right\}$, of expressions

$$
c_{\Lambda}=\sum_{i=1}^{k} n_{t_{i}}\left(f^{t_{i}}\right)-\sum_{i=2}^{k} n_{t_{i-1}}\left(T_{t_{i}}^{t_{i}-1 f^{t_{i}}}\right) .
$$

(We put $c_{\Lambda}=\infty$ if $n_{t_{i}}\left(f^{t_{i}}\right)=\infty$ for some i.) Also $\langle n, f\rangle=\lim c_{\Lambda_{j}}$ for every increasing sequence $\Lambda_{j}$ with the union everywhere dense in $R$.

Theorem 5.1 has been proved in [6] for the case $\langle n, f\rangle=1$ and in [12] for the general case. (An even more general situation has been discussed in [8].) 
5.2. Evidently $\langle n, f\rangle$ is linear in $n$ and $f$. We remark that $\alpha=s_{0}$ a.s. $P_{n}^{f}$ if and only if $n$ is an entrance law at time $s_{0}$, and, in this case,

$$
n_{t}\left(f^{t}\right) \uparrow\langle n, f\rangle \text { as } t \downarrow s_{0} \text {. }
$$

Analogously $\beta=u_{0}$ a.s. $P_{n}^{f}$ if and only if $f$ is an exit law at time $u_{0}$. In this case,

$$
n_{t}\left(f^{t}\right) \uparrow\langle n, f\rangle \text { as } t \uparrow u_{0} \text {. }
$$

A measure $P_{m}^{f}$ corresponding to $m_{t}(B)=p(s, x ; t, B)$ is denoted by $P_{s, x}^{f}$. It follows from (5.5) and (2.5a) that $P_{s, x}^{f}(\Omega)=f^{s}(x)$.

A simple calculation shows that

$$
\langle n, f\rangle=\int_{R} \gamma\left(f^{s}\right) c(s) d s
$$

if $n$ is defined by (4.3), and

$$
\langle n, f\rangle=\int_{R} n_{t}(l) c(t) d t
$$

if $f$ is defined by (4.4).

5.3. With each interval $I$, we associate a sub- $\sigma$-algebra $\mathscr{F}(I)$ of the $\sigma$-algebra $\mathscr{F}$ generated by the sets (5.1) with $t \in I, B \in \mathscr{B}$. We use the following abbreviations

$$
\begin{array}{ll}
\mathscr{F}_{<t}=\mathscr{F}(-\infty, t), & \mathscr{F}_{<t}=\mathscr{F}(-\infty, t], \quad \mathscr{F}_{>t}=\mathscr{F}(t,+\infty), \\
\mathscr{F}_{>t}=\mathscr{F}[t,+\infty), & \mathscr{F}_{<t+}=\bigcap_{u>t} \mathscr{F}_{<u}, \text { etc. }
\end{array}
$$

We put $A \in \mathscr{F}_{\alpha}$ if $\{A, \alpha<t\} \in \mathscr{F}_{<t}$ for all $t \in R$ and $A \in \mathscr{F}_{\beta}$ if $\{A, \beta>t\} \in$ $\mathscr{F}_{>t}$ for all $t \in R$, and we call $\mathscr{F}_{\alpha}$ and $\mathscr{F}_{\beta}$ the germ $\sigma$-algebras at time $\alpha$ and $\beta$.

5.4. We need the following properties of the measure $P_{n}^{f}$.

5.4.A. If $f \in T H, q \in T H^{+}, n \in T M$, then for each $Y \in \mathscr{F}_{<t}$

$$
P_{n}^{f} Y 1_{\alpha<t<\beta}=P_{n}^{q} Y f^{t}\left(x_{t}\right) / q^{t}\left(x_{t}\right)^{4}
$$

If $A \in \mathscr{F}_{<t}$ and $P_{n}^{q}(A)=0$, then $P_{n}^{f}(A)=0$. The same is true for $A \in \mathscr{F}_{\alpha}$.

5.4.B. Let $f \in T H^{+}$. If $X \in \mathscr{F}_{<s}, Y \in \mathscr{F}_{>s}$, then

$$
P_{n}^{f}\left(X 1_{\alpha<s<\beta} Y\right)=P_{n}^{f}\left(X f^{s}\left(x_{s}\right)^{-1} P_{s, x_{s}}^{f} Y\right) .
$$

5.4.C. Let $f \in T H^{+}$. A measure $P$ on $(\Omega, \mathscr{F})$ coincides with one of the measures $P_{n}^{f}, n \in T M$ if and only if

$$
P\left\{x_{t} \in B \mid \mathscr{F}_{<s}\right\}=p^{f}\left(s, x_{s} ; t, B\right) \text { a.s. } P \text { on }\{\alpha<s<\beta\}
$$

for all $s<t \in R$ and all $B \in \mathscr{B}$.

5.4.D. A restriction of a measure $P_{n}^{f}, n \in T M, f \in T H^{+}$, to an arbitrary set $A \in \mathscr{F}_{\alpha}$ is again a measure of the form $P_{\tilde{n}}^{f}$ for some $\tilde{n} \in T M$.

Properties 5.4.A and 5.4.B follow directly from the definition of the measures $P_{n}^{f}$. The necessity of (5.8) follow from 5.4.B and the sufficiency was proved in [6] (see Theorem 3.1). To prove 5.4.D it is sufficient to check that $P(d \omega)=1_{A}(\omega) P_{n}^{f}(d \omega)$ satisfies (5.8). This is easy to do using 5.4.B.

\footnotetext{
${ }^{4}$ We omit the factor $1_{\alpha<t<\beta}$ since $f^{t}\left(x_{t}\right) / q^{t}\left(x_{t}\right)$ is not defined outside the set $\{\alpha<t<\beta\}$.
} 
5.5. The shift $\omega^{\prime}=\theta_{s} \omega$ of the path $\omega$ is given by the formula $\omega^{\prime}(t)=\omega(t+s)$, $\alpha-s<t<\beta-s$. We put $\theta_{s} Y(\omega)=Y\left(\theta_{s} \omega\right)$ for each function $Y(\omega)$. Obviously $\theta_{s} \alpha=\alpha-s, \theta_{s} \beta=\beta-s$ and the $\sigma$-algebras $\mathscr{F}_{\alpha}$ and $\mathscr{F}_{\beta}$ are invariant with respect to $\theta_{s}$.

We put $A \in \mathscr{F}_{\alpha}^{\vartheta}$ if $A \in \mathscr{F}_{\alpha}$ and $\theta_{s} 1_{A}=1_{A}$ a.s. $P_{m}^{f}$ for all $s \in R, m \in M, f \in T H$. Let the transition function $p$ be stationary.

5.5.A. The formulae

$$
\left(k_{s} n\right)_{t}=n_{t+s} \quad\left(k_{s} f\right)^{t}=f^{t+s}
$$

define transformations $k_{s}$ of the classes $T M$ and $T H$, and

$$
P_{n}^{f}\left(\theta_{s} Y\right)=P_{, k_{s} n}^{k_{s} f} Y
$$

for all $Y \in \mathscr{F}$.

5.5.B. A restriction of a measure $P_{m}^{f}, m \in M, f \in T H^{+}$, to a set $A \in \mathscr{P}_{\alpha}^{\oplus}$ is a measure of the form $P_{\tilde{m}}^{f}$ where $\tilde{m} \in M$. If $m$ is a minimal element of $M$, then $P_{m}^{f}$ is trivial on $\mathscr{F}_{\alpha}$ (i.e., each $\mathscr{F}_{\alpha}$-measurable function is constant $P_{m}^{f}$-a.e.).

The statement 5.5.A is an implication of formula (5.2), and 5.5.B follows from 5.4.D, 5.4.A and 5.5.A.

6. Three lemmas.

6.1. In this section we prove three lemmas which make possible the computation of dissipative minimal elements. In the first lemma the behaviour of the ratio of two time-dependent excessive functions along a path is studied. The second one establishes a fundamental identity involving two time-dependent excessive functions and the ratio of their integrals with respect to $t$. The third lemma gives an approximation of the birth time $\alpha$ by stationary stopping times.

6.2. We denote by $T K$ the class of all measures $P_{n}^{f}, n \in T M, f \in T H$. It follows from Subsection 4.6 that the classes $T K$ corresponding to the transition functions $p$ and $p^{q}$ are identical for each $q \in T H^{+}$.

Let $Y_{t}(\omega)$ be a positive function defined for all $\omega \in \Omega, \alpha(\omega)<t<\beta(\omega)$. We say that $Y_{t+}$ is a right $T K$-modification of $Y_{t}$ if $Y_{t+} \in \mathcal{F}_{<t_{+}}$and if, for each countable everywhere dense subset $\Lambda$ of $R$,

$$
Y_{t+}=\lim _{\substack{r \downarrow t \\ s \in \Lambda}} Y_{r} \quad \text { for all } t \in(\alpha, \beta) \text { a.s. } T K .
$$

The left $T K$-modification $Y_{t-}$ of $Y_{t}$ is defined in an analogous way.

LEMMA 6.1. There exist a right TK-modification $Y_{t+}$ and a left $T K$-modification $Y_{t-}$ of the function

$$
Y_{t}=f^{t}\left(x_{t}\right) / q^{t}\left(x_{t}\right)
$$

for every $f \in T H, q \in T H^{+}$. For each $P \in T K$,

$$
Y_{t+}=Y_{t}=Y_{t-} \text { a.s. } P \text { on }\{\alpha<t<\beta\}
$$

for all $t$ except at most a countable set (depending on $P$ ). 
Proof. Fix $\Lambda$ and denote by $N\left(v_{1}, v_{2} ; s, u\right)$ the number of upcrossings of $\left[v_{1}, v_{2}\right]$ by $Y_{t}$ over the set $\Lambda \cap(s, u)$, i.e., the maximal positive integer $k$ such that there exist $s_{1}<u_{1}<\cdots<s_{k}<u_{k} \in \Lambda \cap(s, u)$ with the property $Y_{s_{1}}, \ldots, Y_{s_{k}}<v_{1}$, $Y_{u_{1}}, \ldots, Y_{u_{k}} \geqslant v_{2}$. Put $A(s, u)=\left\{\alpha<s, \beta>u, N\left(v_{1}, v_{2} ; s, u\right)=\infty\right.$ for some $v_{1}$ $\left.<v_{2}\right\}$. The existence of $Y_{t+}$ and $Y_{t-}$ and the equality (6.3) will be proved if we show that $P_{n}^{h} A(s, u)=0$ for all $s<u, h \in T H, n \in T M$ (cf. [1, Theorem 11.2]). By 5.4.A, it suffices to check this only for $h=q$, and since $P_{n}^{q}$ is $\sigma$-finite on the $\sigma$-algebra $\mathscr{F}_{<s}$, it is sufficient to prove that

$$
\int_{A(s, u)} Z P_{n}^{q}(d \omega)=0
$$

for each $P_{n}^{q}$-integrable $Z \in \mathscr{F}_{<s}$. By 5.4.A and (6.2)

$$
P_{n}^{q} Z 1_{\alpha<s} X Y_{t}=P_{n}^{f} Z 1_{\alpha<s, t<\beta} X \text { for all } s<t, X \in \mathscr{F}_{<t} \text {. }
$$

Put $P^{\prime}(d \omega)=1_{\alpha<s} Z P_{n}^{q}(d \omega)$. It follows from (6.5) that $\left(Y_{t}, \mathscr{F}_{<t}, P^{\prime}\right)$ is a supermartingale on $[s, \infty)$. By Doob's inequality

$$
P^{\prime} N\left(v_{1}, v_{2} ; s, u\right)<\left(v_{2}-v_{1}\right)^{-1} P^{\prime}\left(Y_{s}+v_{1}\right)
$$

which means that

$$
P_{n}^{q} Z 1_{\alpha<s} N\left(v_{1}, v_{2} ; s, u\right)<\left(v_{2}-v_{1}\right)^{-1}\left(P_{n}^{f} Z 1_{\alpha<s<\beta}+v_{1} P_{n}^{q} Z 1_{\alpha<s}\right) .
$$

This implies (6.5).

6.3. Now we suppose that the transition function $p$ is stationary. It follows from (5.9) that, if all measures $P$ of $T K$ vanish on $A \in \mathcal{F}$, then they vanish on all sets $\theta_{s} A, s \in R$.

Let

$$
\theta_{s} Y_{t}=Y_{t+s} \text { for all } s \text { and } t
$$

and let $Y_{t+}$ be a right $T K$-modification of $Y_{t}$. Then both $\theta_{s} Y_{t+}$ and $Y_{t+s+}$ are right $T K$-modifications of $Y_{t+s}$. Hence

$$
\theta_{s} Y_{t+}=Y_{t+s+} \quad \text { for all } t \text { a.s. } T K \text {. }
$$

If $m \in M, h \in H$, then, by (5.9), $P_{m}^{h}\left\{Y_{t} \neq Y_{t+}\right\}$ is independent of $t$. It follows from (6.3) that

$$
P_{n}^{h}\left\{Y_{t} \neq Y_{t+}\right\}=0 \text { for all } t .
$$

6.4. A function $\tau(\omega)$ is called a stopping time if $\alpha(\omega)<\tau(\omega)<\beta(\omega)$ for each $\omega$ and $\{\tau<t\} \in \mathscr{F}_{<t}$ for every $t \in R$. If $\theta_{s} \tau=\tau-s$ a.s. $T K$ for all $s \in R$, we say that $\tau$ is stationary.

We put $A \in \mathscr{F}_{\tau}$ if $A \in \mathscr{F}$ and $\{A, \tau<t\} \in \mathscr{F}_{<t}$ for all $t \in R^{\dot{*}}$, and we put $A \in \mathscr{P}_{\tau}^{\oplus}$ if, in addition, $\theta_{s} A=A$ a.s. $T K$ for all $s$.

LemMA 6.2. Let $m \in M, f \in T H^{+}, h \in T H$ and let functions

$$
\bar{f}(x)=\int_{R} f^{t}(x) d t, \quad \bar{h}(x)=\int_{R} h^{t}(x) d t
$$


be finite $m$-a.e. Suppose that $\tau$ is a stationary stopping time, $X \in \mathscr{P}_{\tau}^{9}$ and $Y_{t+}$ is a right TK-modification of

$$
Y_{t}=\bar{h}\left(x_{t}\right) / \bar{f}\left(x_{t}\right)
$$

Then

$$
P_{m}^{f} X Y_{\tau+}=P_{m}^{h} X 1_{\tau<\beta}
$$

Proof. Put

$$
\begin{gathered}
F(\delta)=P_{m}^{f} X \int_{\tau}^{\tau+\delta} Y_{t+} d t, \quad \delta>0 \\
Z_{t}=X 1_{\tau<0<\tau+\delta} Y_{0+} f^{t}\left(x_{0}\right) / \bar{f}\left(x_{0}\right)
\end{gathered}
$$

We have

$$
F(\delta)=\int_{R} P_{m}^{\bar{f}}\left(\theta_{t} Z_{t}\right) d t=\int_{R} P_{m}^{\bar{f}} Z_{t} d t
$$

and, by Fubini's theorem,

$$
F(\delta)=P_{m}^{\tilde{f}} \int_{R} Z_{t} d t=P_{m}^{\bar{f}} X 1_{\tau<0<\tau+\delta} Y_{0+}
$$

By (6.8), $Y_{0+}=Y_{0}$ a.s. $P_{m}^{f}$, and by 5.4.A and (6.9)

$$
F(\delta)=P_{m}^{\bar{h}} X 1_{\tau<0<\tau+\delta}
$$

The right side is independent of $f$. Hence $F(\delta)$ does not change if we replace $f$ by $h$, and, by (6.11), (6.9),

$$
P_{m}^{f} X \int_{\tau}^{\tau+\delta} Y_{t+} d t=P_{m}^{h} X \int_{\tau}^{\tau+\delta} 1_{\alpha<t<\beta} d t
$$

Dividing by $\delta$ and tending $\delta$ to 0 , we get (6.10).

6.5. LEMMA 6.3. If $m \in M$ is dissipative, then there exists a sequence of stationary stopping times $\tau_{n}$ such that

for all $h \in T H$.

$$
\tau_{n} \downarrow \alpha \quad a . s . P_{m}^{h}
$$

Proof. Fix $l \in L_{+}^{1}(m)$ such that $m(l)=1$ and put

$$
\begin{gathered}
q(x)=\int_{0}^{\infty} T_{t} l(x) d t=g_{x}(l), \quad q_{1}(x)=\int_{0}^{\infty} e^{-t} T_{t} l(x) d t, \\
f(x)=g_{x}\left(q_{1}\right) .
\end{gathered}
$$

We have $f, q \in T H^{+}$and $1-f / q=q_{1} / q>0$. Denote by $Y_{t+}$ the right modification of $Y_{t}=f\left(x_{t}\right) / q\left(x_{t}\right)$, and put

$$
a_{u}=\int_{\alpha}^{u \wedge \beta}\left(1-Y_{t+}\right) d t
$$


We prove that

6.5.A. For $P_{m}^{q}$-almost all $\omega$,

(i) $0<a_{u_{1}}<a_{u_{2}}$ for all $\alpha<u_{1}<u_{2}<\beta$,

(ii) $a_{u}<\infty$ for all $\alpha<u<\beta$,

(iii) $a_{u}$ is continuous in $u$ and $a_{u} \downarrow 0$ as $u \downarrow \alpha$.

6.5.B. $a_{u} \in \mathcal{F}_{<u}$.

6.5.C. $\theta_{s} a_{u}=a_{u+s}$ for all $u$ a.s. $T K$.

First, we remark that $q-f=q_{1}>0$ on $E \backslash E_{0}$ and $q<\infty m$-a.e. By Lemma 6.1 there exists an at most countable set $\Delta$ such that

$$
1-Y_{t+}=1-Y_{t}=q_{1}\left(x_{t}\right) / q\left(x_{t}\right)>0 \text { a.s. } P_{m}^{q} \text { on }\{\alpha<t<\beta\} \text { for each } t \bar{\in} \Delta
$$

which implies 7.1.A, (i). We have

where

$$
P_{m}^{q} a_{u} \frac{q_{1}\left(x_{u}\right)}{q\left(x_{u}\right)}=\int_{0}^{\infty} F(s) d s
$$

$$
F(s)=P_{m}^{q} \frac{q_{1}\left(x_{u-s}\right)}{q\left(x_{u-s}\right)} \frac{q_{1}\left(x_{u}\right)}{q\left(x_{u}\right)}=m\left(\frac{q_{1}}{s} T_{s} q_{1}\right)
$$

Therefore

$$
P_{m}^{q} a_{u} \frac{q_{1}\left(x_{u}\right)}{q\left(x_{u}\right)}=m\left(\frac{q_{1} f}{q}\right)<m\left(q_{1}\right)<m(l)=1 .
$$

Taking into account (6.13), we have 6.5.A, (ii). The property (iii) is an obvious implication of (ii), and 6.5.B, $\mathrm{C}$ follow from the fact that $Y_{t+} \in \mathcal{F}_{<t+}$ and (6.7).

For each $\varepsilon>0$, we set

$$
\tau_{\varepsilon}=\inf \left\{t: a_{t}>\varepsilon\right\}, \quad \tau_{\varepsilon}=\beta \text { if } a_{t}<\varepsilon \text { for all } t .
$$

This is a stationary stopping time. Indeed, $\left\{\tau_{\varepsilon}<t\right\}=\left\{a_{t}>\varepsilon\right\} \cup\{\beta<t\} \in \mathscr{F}_{<t}$ by 6.5.B, and $\theta_{s} \tau_{e}=\tau_{\varepsilon}-s$ a.s. $T K$ by 6.5.C. If $\varepsilon_{n} \downarrow 0$, then $\tau_{\varepsilon} \downarrow \alpha$ a.s. $P_{m}^{q}$, and (6.12) follows from 5.4.A.

\section{Dissipative minimal elements.}

7.1. TheOREM 7.1. Let $m$ be a dissipative element of $M, f \in \mathrm{TH}^{+}, h \in \mathrm{TH}$ and let $\langle m, f\rangle\langle\infty,\langle m, h\rangle\langle\infty$. Suppose that the functions

$$
\bar{f}(x)=\int_{R} f^{t}(x) d t, \quad \bar{h}(x)=\int_{R} h^{t}(x) d t
$$

are finite $m$-a.e. Then there exists a function $Y_{\alpha+} \in \mathscr{P}_{\alpha}^{9}$ such that

$$
Y_{\alpha+}=\lim _{\substack{s \downarrow \alpha \\ r \in \Lambda}}\left(\bar{h}\left(x_{r}\right) / \bar{f}\left(x_{r}\right)\right) \quad \text { a.s. } P_{m}^{q}
$$

for each countable everywhere dense subset $\Lambda$ of $R$ and each $q \in T H$. Moreover

$$
P_{m}^{f} X Y_{\alpha+}=P_{m}^{h} X \text { for all } X \in \mathscr{P}_{\alpha}^{\theta}
$$

and, if $m$ is minimal, then

$$
Y_{\alpha+}=\langle m, h\rangle /\langle m, f\rangle \quad \text { a.s. } P_{m}^{f} .
$$


Proof. If $\tau$ is a stationary stopping time, then so are $\tau+\boldsymbol{u}$ for all $\boldsymbol{u}>0$. By Lemma 6.2, if $s<t, X \in \mathscr{P}_{\tau+s}^{\oplus}$, then

$$
P_{m}^{f} X Y_{\tau+t+}=P_{m}^{h} X 1_{\tau+t<\beta}<P_{m}^{h} X 1_{\tau+s<\beta}=P_{m}^{f} X Y_{\tau+s+}
$$

where $Y_{t}$ is defined by (6.9). Hence

$$
\left(Y_{\tau+t+}, \mathscr{F}_{\tau+t+}^{\oplus}, P_{m}^{f}\right)_{t>0}
$$

is a supermartingale. It follows from (6.10) that $P_{m}^{f} Y_{\tau+}\left\langle\langle m, h\rangle\right.$. Let $N_{\tau}$ be the number of upcrossings of $\left[v_{1}, v_{2}\right]$ by $Y_{t+} \operatorname{over}(\tau, \infty)$. Since $Y_{t+}$ is right-continuous in $t$ a.s. $P_{m}^{f}$, we have

$$
P_{m}^{f} N_{\tau} \leqslant\left(v_{2}-v_{1}\right)^{-1}\left(\langle m, h\rangle+v_{1}\langle m, f\rangle\right) .
$$

Applying this inequality to the sequence $\tau_{n}$ and passing to the limit, we get the inequality $P_{m}^{f} N_{\alpha}<\infty$. Put $\Omega^{\prime}=\left\{N_{\alpha}<\infty\right\}, \Omega^{\prime \prime}=\Omega \backslash \Omega^{\prime}$. Obviously $\Omega^{\prime}$ and $\Omega^{\prime \prime}$ belong to $\mathscr{F}_{\alpha}^{\theta}$ and $P_{m}^{f}\left(\Omega^{\prime \prime}\right)=0$. Put $Y_{\alpha+}=\lim _{t \downarrow \alpha} Y_{t+}$ on $\Omega^{\prime}, Y_{\alpha+}=0$ on $\Omega^{\prime \prime}$. Then $Y_{\alpha+} \in \mathscr{F}_{\alpha}$ and (7.2) holds for $q=f$. By 5.4.A it holds for all $q \in T H$.

By Fatou's lemma, it follows from (6.10) that

$$
P_{m}^{f} Y_{\alpha+}\left\langle\lim P_{m}^{f} Y_{\tau_{n}+}=\lim P_{m}^{h} 1_{\tau_{n}<\beta}=\langle m, h\rangle<\infty .\right.
$$

Hence $Y_{\alpha+}<\infty$ a.s. $P_{m}^{f}$ and, by 5.4.A, $Y_{\alpha+}<\infty$ a.s. $P_{m}^{h}$ as well. Thus, for each $\varepsilon>0$, there exists $c_{\varepsilon}$ such that $P_{m}^{h}\left\{Y_{\alpha}>c_{\varepsilon}\right\}<\varepsilon$, and, by (6.10)

$$
P_{m}^{f} 1_{Y_{\alpha+}>c_{\varepsilon}} Y_{\tau_{n}+}<P_{m}^{h}\left\{Y_{\alpha+}>c_{\varepsilon}\right\}<\varepsilon .
$$

Hence $Y_{\tau_{n}}$ are uniformly integrable relative to $P_{m}^{f}$ and (6.10) implies (7.3).

If $m$ is minimal, then, by 5.5.B, there exists a constant $C$ such that $Y_{\alpha+}=C$ a.s. $P_{m}^{f}$. We have $P_{m}^{f} Y_{\alpha+}=C P_{m}^{f}(\Omega)=C\langle m, f\rangle$ and, by (7.3), $C=\langle m, h\rangle /\langle m, f\rangle$. This proves (7.4).

7.2. We apply Theorem 7.1 to the excessive functions

$$
h^{s}(x)=\int p(s, x ; t, d y) \varphi(y)=T_{t-s} \varphi(x)
$$

and

$$
f^{s}(x)=\int_{s}^{\infty} d t c(t) \int p(s, x ; t, d y) \psi(y)=\int_{0}^{\infty} T_{u} \psi(x) c(s-u) d u
$$

where $\psi>0, c(t)>0$ and $\int_{R} c(t) d t=1$. Obviously $f \in \mathrm{TH}^{+}$,

$$
\langle m, f\rangle=m(\psi), \quad\langle m, h\rangle=m(\varphi), \quad \bar{f}(x)=g_{x}(\psi), \quad \bar{h}(x)=g_{x}(\varphi) .
$$

Hence, in this case,

$$
Y_{\alpha+}=\lim _{\substack{r \downarrow \alpha \\ r \in \Lambda}}\left(g_{x_{r}}(\varphi) / g_{x_{r}}(\psi)\right) \text { a.s. } P_{m}^{q}
$$

and we arrive at the following result.

THEOREM 7.2. Let $m$ be a dissipative minimal element of $M$. Then for all $\varphi \in L^{1}(m), \psi \in L_{+}^{1}(m), q \in T H$

$$
m(\varphi) / m(\psi)=\lim _{\substack{r \downarrow \alpha \\ r \in \Lambda}}\left(g_{x_{r}}(\varphi) / g_{x_{r}}(\psi)\right) \text { a.s. } P_{m}^{q} .
$$


(The meaning of $\Lambda$ is the same as in Theorem 7.1.)

\subsection{Corollary. If}

$$
m=\int_{R} \nu_{s} d s
$$

where $\nu \in T M$, then (7.8) holds a.s. $P_{\nu}^{q}$.

Indeed, the relation (7.9) implies that $m=\int_{R} k_{s} \nu d s$. Let $\Omega^{\prime}$ and $\Omega^{\prime \prime}$ be the sets defined in the proof of Theorem 7.1. We have

$$
0=P_{m}^{f}\left(\Omega^{\prime \prime}\right)=\int_{R} P_{k_{s} \nu}^{f}\left(\Omega^{\prime \prime}\right) d s
$$

and, by (5.9),

$$
P_{k_{s} \nu}^{f}\left(\Omega^{\prime \prime}\right)=P_{\nu}^{f}\left(\theta_{s} \Omega^{\prime \prime}\right)=P_{\nu}^{f}\left(\Omega^{\prime \prime}\right)
$$

Hence $P_{\nu}^{f}\left(\Omega^{\prime \prime}\right)=0$, and (7.8) holds a.s. $P_{\nu}^{f}$. By 5.4.A, it holds a.s. $P_{\nu}^{q}$.

REMARK. It has been proved in [6] that each $m \in T M$ has an integral representation $m_{s}(B)=\int n_{s}(B) \mu(d n)$ where $\mu$ is a finite measure on the space of minimal elements of $T M$ (which are entrance laws). It has been proved also that if $m$ is a minimal element of $M$ and if $l>0, m(l)=1$, then for each $\varphi \in L^{1}(m)$ and $\mu$-almost all $n$

$$
n_{I}(\varphi) / n_{I}(l) \rightarrow m(\varphi) \quad \text { as } I \uparrow R
$$

where $n_{I}(\varphi)$ means the integral of $n_{s}(\varphi)$ over a finite interval $I$. If $m$ is a null-excessive element (i.e., if $m T_{t} \rightarrow 0$ as $t \rightarrow \infty$ ), then $\mu$ is concentrated on the entrance laws at finite times and $n_{R}(l)<\infty$. Hence $m(\varphi)=n_{R}(\varphi) / n_{R}(l)$, and (7.9) holds with $\nu_{s}=n_{R}(l)^{-1} n_{s}$. It holds also for all entrance laws $k_{u} \nu$. Thus (7.8) is satisfied a.s. $P_{\nu}^{q}$ for some entrance law $\nu$ at time 0 . This justifies the construction described in Subsection 2.7.

7.4. To investigate dissipative elements of $H$ we introduce a backward transition function

$$
\hat{p}(s, d x ; t, y)=\gamma(d x) \rho_{t-s}(x, y)
$$

and we denote by $T \hat{M}, T \hat{H}$ the corresponding classes of time dependent excessive measures and functions. The notations $\hat{M}, \hat{N}, T \hat{M}^{+}$have an analogous meaning. To each $m \in T \hat{M}, f \in T \hat{H}$ there corresponds a measure $\hat{P}_{m}^{f}$, and we set $\langle m, f\rangle^{\prime}=$ $\hat{P}_{m}^{f}(\Omega)$.

Considering reversed time direction, we get the following version of Theorem 7.1.

THEOREM 7.3. Let $m$ be a dissipative minimal element of $\hat{M}$ and let $f \in T \hat{H}^{+}$, $q \in T \hat{H},\langle m, f\rangle^{\prime}\left\langle\infty,\langle m, q\rangle^{\prime}\langle\infty\right.$,

$$
\bar{f}(y)=\int_{R} f^{s}(y) d s<\infty, \quad \bar{q}(y)=\int_{R} q^{s}(y) d s<\infty \quad \text { m-a.e. }
$$

Then there exists a function $Y_{\beta-} \in \mathscr{F}_{\beta}^{9}$ such that

$$
Y_{\beta-}=\lim _{\substack{r \uparrow \beta \\ r \in \Lambda}}\left(\bar{q}\left(x_{r}\right) / \bar{f}\left(x_{r}\right)\right) \text { a.s. } \hat{P}_{m}^{f}
$$


for each countable everywhere dense $\Lambda$. Moreover

$$
\hat{P}_{m}^{f} X Y_{\beta-}=\hat{P}_{m}^{q} X \quad \text { for all } X \in \mathscr{F}_{\beta}^{9}
$$

and, if $m$ is minimal, then

$$
Y_{\beta-}=\langle m, q\rangle^{\prime} /\langle m, f\rangle^{\prime} \quad \text { a.s. } \hat{P}_{m}^{f} .
$$

7.5. The following result follows immediately from Theorem 7.3.

THEOREM 7.4. Let $h$ be a dissipative minimal element of $H$. If $f \in T M$ and if $h$ is integrable with respect to measures $\xi$ and $\eta$, then

$$
\xi(h) / \eta(h)=\lim _{\substack{r \uparrow \beta \\ r \in \Lambda}}\left(\xi\left(g^{x_{r}}\right) / \eta\left(g^{x_{r}}\right)\right) \text { a.s. } P_{\gamma^{f .}}^{h}
$$

To prove this statement, we apply Theorem 7.3 to the measure $m(d x)=\gamma^{h}(d x)$ $=h(x) \gamma(d x)$ which, according to Subsection 3.5 , is a minimal and dissipative element of $\hat{M}$, and to the functions

$$
q^{s}(y)=\int \xi(d x) \rho_{s}(x, y), \quad f^{s}(y)=\int_{0}^{\infty} c(s+u) d u \int \eta(d x) \rho_{u}(x, y) .
$$

By simple computations we get the formulae

$$
\left\langle\gamma^{h}, q\right\rangle=\xi(h), \quad\left\langle\gamma^{h}, f\right\rangle=\eta(h), \quad \bar{q}(y)=\xi\left(g^{y}\right), \quad \bar{f}(y)=\eta\left(g^{y}\right)
$$

and we notice that the measure $\hat{P}_{\gamma^{h}}^{f}$ corresponding to $\hat{p}, \gamma^{h}, f$ coincides with the measure $P_{\gamma^{f}}^{h}$.

7.6. As in Subsection 7.3, we prove that, if

$$
h=\int_{R} \varphi^{t} d t
$$

where $\varphi \in T H$, then (7.11) is fulfilled a.s. $P_{\gamma^{\prime}}^{\varphi}$. If $h$ is null-excessive, then (7.12) holds for some exit law at time 0 .

\section{Appendix. Decomposition into minimal elements.}

0.1 . Let $p_{t}(x, B)$ be a stationary transitive function in a standard Borel space $(E, \mathscr{B})$ and let $M$ and $H$ be the corresponding classes of excessive measures and functions. For every $l \in \mathscr{B}$, we put $M^{l}=\{m: m \in M, m(l)=1\}$ and we denote by $\mathscr{B}\left(M^{l}\right)$ the $\sigma$-algebra in $M^{l}$ generated by the sets $\left\{m: m \in M^{l}, m(B)<u\right\}$, $B \in \mathscr{B}, u \in R$. For every measure $\nu$ on $\mathscr{B}$, we put $H^{\nu}=\{h: h \in H, \nu(h)=1\}$ and we denote by $\mathscr{B}\left(H^{\nu}\right)$ the $\sigma$-algebra in $H^{\nu}$ generated by the sets $\left\{h: h \in H^{\nu}\right.$, $\xi(h)<u\}, \xi$ is a measure on $\Re, u \in R$. Our objective is to prove the following two results.

Theorem 0.1. Let $\Gamma$ be the set of all minimal elements of $M$ which belong to $M^{\prime}$. Suppose that $l \in \mathscr{B}$ is strictly positive. Then $\Gamma \in \mathscr{B}\left(M^{l}\right)$ and, for each $m \in M^{l}$, there exists one and only one probability measure $\mu$ on $\mathscr{B}\left(M^{l}\right)$ concentrated on $\Gamma$ such that

$$
m(B)=\int_{\Gamma} n(B) \mu(d n) \text { for all } B \in \mathscr{B}
$$


TheOREM 0.2. Let $\Gamma$ be the set of all minimal elements of $H$ belonging to $H^{\nu}$. Let $\nu$ be a $\sigma$-finite measure with the property:

0.1.A. There exists an excessive reference measure $\gamma$ and a strictly positive function l such that

$$
\gamma(l h) \leqslant \nu(h) \text { for all } h \in H .
$$

Then $\Gamma \in \mathscr{B}\left(H^{\nu}\right)$ and, for every $h \in H^{\nu}$, there exists one and only one probability measure $\mu$ on $\mathscr{B}\left(H^{\nu}\right)$ concentrated on $\Gamma$ such that

$$
h(x)=\int_{\Gamma} f(x) \mu(d f) \text { for all } x \in B
$$

0.2. Comments to Theorem 0.2 .

(a) If $\gamma$ is a reference measure and if $h \in H$, then $h<\infty$ a.s. $\gamma$. If $h \neq 0$, then $\gamma(h)>0$ and $\gamma(l h)=1$ for some strictly positive function $l$. The measure $\nu(d x)=$ $l(x) \gamma(d x)$ obviously satisfies 0.1 .A, and $h \in H^{\nu}$. Hence each $h \in H$ can be decomposed into minimal elements. (The analogous statement for $m \in M$ is obvious.)

(b) Let $\gamma$ satisfy condition 2.2.C and let $g^{y}$ be the corresponding Green function defined by (2.14). Suppose that the function $q(y)=\nu\left(g^{y}\right)$ is finite $\gamma$-a.e. and strictly positive. Then the measure $\nu$ has property 0.1 .A. In fact,

$$
\gamma_{1}(B)=\int_{E} \nu(d x) g_{x}(B)=\int_{B} q(y) \gamma(d y)
$$

is an excessive reference measure and $\gamma_{1}(l h) \leqslant \nu(h)$ for all $h \in H$ where

$$
l(y)=q(y)^{-1} \int_{0}^{\infty} d t e^{-t} \int_{E} \nu(d x) \rho_{t}(x, y)
$$

is strictly positive.

(c) In case of Brownian motion remark (b) is applicable to Lebesgue measure $\gamma$ and any measure $\nu$ concentrated at one point.

(d) If $\gamma$ is a reference measure, the $\sigma$-algebra $\mathscr{B}\left(H^{\nu}\right)$ is generated by the sets $\left\{h: h \in H^{\nu}, \gamma(l h)<u\right\}, l \in \mathscr{B}, u \in R$. This follows from the relation

$$
\xi(h)=\lim _{t \downarrow 0} \gamma\left(l_{t} h\right) \quad \text { where } l_{t}(y)=\int \xi(d x) \rho_{t}(x, y) .
$$

(e) The function $f(x), f \in H, x \in E$ is $\mathscr{B}\left(H^{\nu}\right) \times \mathscr{B}$-measurable. In fact, it is easy to see that $\int r(x, y) \gamma(d y) f(y)$ is $\mathscr{B}\left(H^{\nu}\right) \times \mathscr{B}$-measurable if $r(x, y)$ is $\mathscr{B} \times \mathscr{B}$ measurable, and measurability of $f(x)$ follows from the formula $f(x)=$ $\lim _{t \downarrow 0} \int \rho_{t}(x, y) \gamma(d y) f(y)$.

(f) By remark (e) and the Fubini theorem, it follows from (0.3) that $\xi(h)=$ $\int_{\Gamma} \xi(f) \mu(d f)$ for each measure $\xi$.

0.3 . Theorem 0.2 follows easily from Theorem 0.1 . First, if $\nu(d x)=l(x) \gamma(d x)$ where $\gamma$ is an excessive reference measure and $l>0$, then the mapping (3.20) establishes a 1-1 correspondence between $H^{\nu}$ and $\hat{M}^{l}$ (we use the notations of Subsection 3.5). Obviously this mapping is measurable. By 0.2.d the inverse mapping is also measurable, and the representation $(0.1)$ of the measure $m=\gamma^{h} \in$ $\hat{M}^{l}$ is equivalent to the representation (0.3) of $h \in H^{\nu}$. 
Now let $\nu$ satisfy condition 0.1.A and let $\nu_{1}(d x)=l(x) \gamma(d x)$. We note that $0<\nu_{1}(h)<1$ for all $h \in H^{\nu}$. Put $H_{0}^{\nu_{1}}=\left\{h: h \in H^{\nu_{1}}, \nu(h)<\infty\right\}$. Formulae $F_{1}(h)=h / \nu_{1}(h)$ and $F(h)=h / \nu(h)$ determine inverse measurable mappings $F_{1}$ of $H^{\nu}$ onto $H_{0}^{\nu_{1}}$ and $F_{2}$ of $H_{0}^{\nu_{1}}$ onto $H^{\nu}$. Evidently they preserve minimal elements. Therefore to prove Theorem 0.2 for the measure $\nu$, it is sufficient to check that each element $h \in H_{0}^{\nu_{1}}$ has a unique integral representation through minimal elements of $H$ which lie in $H_{0}^{\nu_{1}}$. Since Theorem 0.2 has been proved for the measure $\nu_{1}$, there corresponds to every $h \in H^{\nu_{1}}$ a unique probability measure $\mu$ on $\mathscr{B}\left(H^{\nu_{1}}\right)$ concentrated on the set $\Gamma_{1}$ of minimal elements such that

$$
h(x)=\int_{\Gamma_{1}} f(x) \mu(d f)
$$

By 0.2.f this implies an equality

$$
\nu(h)=\int_{\Gamma_{1}} \nu(f) \mu(d f)
$$

and since $\nu(h)<\infty$, the measure $\mu$ is concentrated on $\Gamma_{1} \cap H_{0}^{\nu_{1}}$.

0.4. The rest of the Appendix is devoted to proving the following statement.

THEOREM 0.3. Let $l>0$ and let

$$
f^{s}(x)=\int_{0}^{\infty} T_{u} l(x) c(s-u) d s
$$

where $c(t)>0$ and $\int_{R} c(t) d t=1$. Let $\mathscr{F}_{\alpha}^{9}$ be the $\sigma$-algebra defined in Subsection 5.5. There exists an $M^{l}$-valued function $n^{\omega}$ on $\Omega$ with the properties:

0.4.A. $n^{\omega}(B)$ is $\mathscr{F}$-measurable for every $B \in \mathscr{B}$.

0.4.B. $P_{m}^{f}\left\{Z \mid \mathscr{P}_{\alpha}^{\oplus}\right\}=P_{n^{\omega}}^{f} Z$ a.s. $P_{m}^{f}$ for all $Z \in \mathscr{F}, m \in M^{l}$.

Properties 0.4.A, B mean that $\mathscr{P}_{\alpha}^{\theta}$ is an $H$-sufficient $\sigma$-algebra for the class $K$ of probability measures $P_{m}^{f}, m \in M^{l}$, and Theorem 0.1 follows from Theorem 0.3 because of the general relation between $H$-sufficient statistics and minimal elements established in [7] (see Theorem 3.1).

0.5. In each standard Borel space $\left(E, \mathscr{B}^{3}\right)$ there exists a support system $W$ i.e., a countable family of positive bounded functions with the properties:

0.5.A. If $\mu_{n}$ is a sequence of probability measures on $\mathscr{B}$ and if $\lim \mu_{n}(\varphi)=q(\varphi)$ exists for every $\varphi \in W$, then there is a probability measure $\mu$ such that $\mu(\varphi)=q(\varphi)$ for all $\varphi \in W$.

0.5.B. If a class $\mathcal{S}$ of positive functions contains $W$ and is closed under addition, multiplication by positive constants and if $\delta$ contains together with each increasing sequence its limit, then $\delta$ contains all functions $\varphi \in \mathscr{B}$.

We put $x \in E^{\prime}$ if the limit

$$
\lim _{u \rightarrow \infty}\left(g_{x}^{u}(\varphi l) / g_{x}^{u}(l)\right)
$$

exists for all $\varphi \in W$. By 0.5.A for each $x \in E^{\prime}$ there exists a probability measure $\mu_{x}$ such that the limit $(0.5)$ coincides with $\mu_{x}(\varphi)$. We put $\varphi^{l}=\varphi^{-1}, n_{x}(d y)=$ $\mu_{x}(d y) l^{-1}(y)$. Obviously $n_{x}(l)=1$ and

$$
\lim _{u \rightarrow \infty}\left(g_{x}^{u}(\varphi) / g_{x}^{u}(l)\right)=n_{x}(\varphi) \text { if } x \in E^{\prime}, \varphi^{l} \in W
$$


Let $\Lambda_{0}$ be the set of all rational numbers. We put $\omega \in \Omega^{\prime}$ if $x_{r} \in E^{\prime}$ for all $r \in \Lambda_{0}$ and if

$$
\lim _{\substack{r \downarrow \alpha \\ r \in \Lambda_{0}}} n_{x_{r}}(\varphi)
$$

exists for all $\varphi^{l} \in W$. Again by 0.5.A, for every $\omega \in \Omega^{\prime}$, there exists a measure $n^{\omega}(\varphi)$ such that $n^{\omega}(l)=1$ and

$$
n^{\omega}(\varphi)=\lim _{\substack{r \downarrow \alpha \\ r \in \Lambda_{0}}} n_{x_{r}}(\varphi) \text { for all } \omega \in \Omega^{\prime}, \varphi^{l} \in W .
$$

We shall see that $E^{\prime}$ and $\Omega^{\prime}$ are not empty (except the case where $M^{l}$ is empty, in which case our theorem is trivial). Let

$$
E^{\prime \prime}=E \backslash E^{\prime}, \quad \Omega^{\prime \prime}=\Omega \backslash \Omega^{\prime} .
$$

Fix arbitrary points $x^{\prime} \in E^{\prime}$ and $\omega^{\prime} \in \Omega^{\prime}$ and put $n_{x}=n_{x^{\prime}}$ for $x \in E^{\prime \prime}, n^{\omega}=n^{\omega^{\prime}}$ for $\omega \in \Omega^{\prime \prime}$.

The function $n^{\omega}(\varphi)$ is $\mathscr{F}$-measurable if $\varphi^{l} \in M$. By 0.5.B, the same is true for all $\varphi \in \mathscr{B}$. Therefore $n^{\omega}$ satisfies the condition 0.4 .A. Theorem 0.3 will be proved if we show that, for each $m \in M^{l}$,

$$
P_{m}^{f}\left\{n^{\omega} \bar{\in} M=0\right.
$$

and 0.4.B is fulfilled. We check this separately for dissipative and conservative $m$.

0.6. Suppose that $m \in M^{l}$ is dissipative. Obviously (0.6) holds with

$$
n_{x}(\varphi)=g_{x}(\varphi) / g_{x}(l)
$$

if $g_{x}(l)<\infty$. Hence $E^{\prime \prime} \subset\left\{x: g_{x}(l)=\infty\right\}$ and $m\left(E^{\prime \prime}\right)=0$.

Let $\varphi^{\prime} \in W$. Comparing (0.7), (0.8) and (7.7) and applying Theorem 7.1, we conclude that $P_{m}^{f}\left(\Omega^{\prime \prime}\right)=0$, that the function $n^{\omega}(\varphi)$ is $\mathscr{P}_{\alpha}^{\oplus}$-measurable and that

$$
P_{m}^{f} X n^{\omega}(\varphi)=P_{m}^{h} X
$$

with $h^{s}(x)=T_{t-s} \varphi(x)$ for all $X \in \mathscr{F}_{\alpha}^{9}, t \in R$. By 5.4.B and 5.4.A, for $s<t$,

$$
\begin{aligned}
P_{m}^{f}\left[X 1_{\alpha<s} f^{t}\left(x_{t}\right)^{-1} \varphi\left(x_{t}\right)\right] & =P_{m}^{f}\left[X f^{s}\left(x_{s}\right)^{-1} P_{s, x_{s}}^{f}\left(\varphi\left(x_{t}\right) f^{t}\left(x_{t}\right)^{-1}\right)\right] \\
& =P_{m}^{f}\left[X f^{s}\left(x_{s}\right)^{-1} h^{s}\left(x_{s}\right)\right]=P_{m}^{h}\left(X 1_{\alpha<s<\beta}\right) .
\end{aligned}
$$

Since $\beta=t$ a.s. $P_{m}^{h}$, we get by setting $s \uparrow t$

$$
P_{m}^{f}\left[X f^{t}\left(x_{t}\right)^{-1} \varphi\left(x_{t}\right)\right]=P_{m}^{h} X .
$$

It follows from $(0.10)$ and $(0.11)$ that

$$
P_{m}^{f} f^{t}\left(x_{t}\right)^{-1} \varphi\left(x_{t}\right) X=P_{m}^{f} X n^{\omega}(\varphi)
$$

Established for $\varphi^{l} \in W$, equality (0.12) can be extended for all $\varphi \in \mathscr{B}$ using 0.5.B. Suppose that

$$
\varphi(x)=P_{t, x}^{f} Z, \quad Z \in \mathscr{F}_{>t} .
$$

Since $X 1_{\alpha<t} \in \mathscr{F}_{<t}$, formula (0.12) and 5.4.B imply that

$$
P_{m}^{f} X n^{\omega}(\varphi)=P_{m}^{f} X 1_{\alpha<t<\beta} Z .
$$


We apply this formula to $t=-u<0$ and $Z=\psi\left(x_{0}\right) / f^{0}\left(x_{0}\right)$ and we get

$$
P_{m}^{f} X n^{\omega}\left(T_{u} \psi\right)=P_{m}^{f} X 1_{\alpha<-u} \frac{\psi\left(x_{0}\right)}{f^{0}\left(x_{0}\right)}
$$

On the other hand, it follows from (0.12) that

$$
P_{m}^{f} X n^{\omega}(\psi)=P_{m}^{f} X \psi\left(x_{0}\right) / f^{0}\left(x_{0}\right)
$$

Comparing (0.15) and (0.16) we have

$$
n^{\omega}\left(T_{u} \psi\right) \leqslant n^{\omega}(\psi) \text { a.s. } P_{m}^{f}
$$

and

$$
n^{\omega}\left(T_{1 / k} \psi\right) \rightarrow n^{\omega}(\psi) \text { a.s. } P_{m}^{f} \text {. }
$$

The set $C=\left\{(u, \omega): n^{\omega}\left(T_{u} \psi\right)>n^{\omega}(\psi)\right.$ for some $\left.\psi^{l} \in W\right\}$ is $\mathscr{B}_{R} \times \mathscr{F}$ measurable and all its $u$-sections have $P_{m}^{f}$ measure zero. By Fubini's theorem, there exists a set $\Omega_{0} \in \mathcal{F}$ such that $P_{m}^{f}\left(\Omega \backslash \Omega_{0}\right)=0$ and $(u, \omega) \bar{\in} C$ for almost all $u$ and all $\omega \in \Omega_{0}$. Using 0.5.B and the semigroup property of $T_{t}$, we prove that $n^{\omega}\left(T_{u} \psi\right)<$ $n^{\omega}(\psi)$ for all $\omega \in \Omega_{0}$ and all $u \in R$. Because of (0.18), there exists a set $\Omega_{1} \subset \Omega_{0}$ such that $P_{m}^{f}\left(\Omega \backslash \Omega_{1}\right)=0$ and $n^{\omega}\left(T_{1 / k} \psi\right) \rightarrow n^{\omega}(\psi)$ for all $\omega \in \Omega_{1}$ and all $\psi^{l} \in W$. Applying 0.5.A and 0.5.B, it is easy to prove that $n^{\omega} \in M$ for all $\omega \in \Omega_{1}$.

To prove 0.4.B, we establish that, for each $Z \in \mathcal{F}$,

(i) $P_{n^{\omega}}^{f} Z$ coincides $P_{m}^{f}$-a.e. with an $\mathscr{F}_{\alpha}$-measurable function,

(ii) $P_{m}^{f} X Z=P_{m}^{f}\left(X P_{n}^{f}{ }^{\omega} Z\right)$ for all $X \in \mathscr{F}_{\alpha}^{9}$.

It is sufficient to check this for $Z=1_{\alpha<t} f\left(x_{t_{1}}, \ldots, x_{t_{n}}\right) 1_{u<\beta}$ where $t<t_{1}$ $<\cdots<t_{n}<u$ and $f$ is a measurable function on $E^{n}$. It follows from (5.2) that in this case $P_{n^{\omega}}^{f} Z=n^{\omega}(\varphi)$ where $\varphi$ is given by $(0.13)$. Both conditions (i) and (ii) are satisfied (the second one follows from (0.14)).

0.7. Now let $m$ be a conservative element of $M^{l}$. Then by Theorem 3.2, $m\left(E^{\prime \prime}\right)=0$ and $(0.6)$ holds with

$$
n_{x}(\varphi)=m^{l}\left(\frac{\varphi}{l} \mid \mathscr{B}_{m}^{t}\right), \quad m \text {-a.e. }
$$

The following two lemmas establish relations between the $\sigma$-algebra $\mathscr{B}_{m}^{T}$ and $\mathscr{F}_{\alpha}$.

LEMMA 0.1. If $F$ is a bounded $\mathscr{B}_{m}^{T}$-measurable function, then there exists a $\mathscr{F}_{\alpha}$-measurable function $Y_{F}$ such that, for each countable everywhere dense set $\Lambda$,

$$
Y_{F}=\lim _{\substack{r \downarrow-\infty \\ r \in \Lambda}} F\left(x_{r}\right) .
$$

For each $F \in \mathscr{B}_{m}^{T}$ and each $\varphi \in \mathscr{B}, t \in R$

$$
P_{m}^{f} Y_{F} n^{\omega}(\varphi)=P_{m}^{f} Y_{F} \varphi\left(x_{t}\right) f^{t}\left(x_{t}\right)^{-1}
$$

Proof. Let $Z$ and $\varphi$ be as in (0.13). By 5.4.B and (5.2), we have for $s<t$, $\psi \in \mathscr{B}$,

$$
P_{m}^{f} F\left(x_{s}\right) \psi\left(x_{t}\right) Z=P_{m}^{f} F\left(x_{s}\right) \psi\left(x_{t}\right) \varphi\left(x_{t}\right) f^{t}\left(x_{t}\right)^{-1}=m\left[F T_{t-s}(\psi \varphi)\right]
$$

Analogously

$$
P_{m}^{f} F\left(x_{t}\right) \psi\left(x_{t}\right) Z=m(F \psi \varphi)
$$


Since $F \in \mathscr{B}_{m}^{T}$, the terms on the right side of $(0.22)$ and $(0.23)$ coincide. This implies the relation

$$
P_{m}^{f}\left\{F\left(x_{s}\right) \mid \mathscr{F}_{>t}\right\}=F\left(x_{t}\right) \text { a.s. } P_{m}^{f} \text { on }\{\beta>t\} .
$$

Hence, for each $u,\left(F\left(x_{t}\right) 1_{\beta>u}, \mathscr{F}_{>t}, P_{m}^{f}\right)$ is a martingale on $(-\infty, u)$ and the existence of $Y_{F}$ follows from the theorem on convergence of a bounded martingale. By (0.19) and (3.8)

$$
\int m(d x) F(x) n_{x}(\varphi) T_{u} l(x)=\int m(d x) n_{x}(\varphi) l(x) F(x)=m(F \varphi)=m\left(F T_{t-r} \varphi\right) .
$$

Therefore, by (0.4)

$$
\begin{aligned}
P_{m}^{f} F\left(x_{r}\right) n_{x_{r}}(\varphi) & =\int m(d x) F(x) n_{x}(\varphi) f^{r}(x)=m\left(F T_{t-r} \varphi\right) \\
& =P_{m}^{f} F\left(x_{r}\right) \varphi\left(x_{t}\right) f^{t}\left(x_{t}\right)^{-1} .
\end{aligned}
$$

Letting $r \rightarrow-\infty$, we get $(0.21)$, first, for $\varphi^{l} \in W$ and then, using 0.5.B, for all $\varphi \in \Re$.

LEMMA 0.2. Every bounded function $Y \in \mathscr{P}_{\alpha}^{P}$ coincides $P_{m}^{f}$-a.e. with $Y_{F}$ for some $F \in \mathscr{B}_{m}^{T}$.

Proof. We choose a function $F \in \mathscr{B}$ such that $P_{m}^{f}\left\{Y \mid x_{0}\right\}=F\left(x_{0}\right)$ a.s. $P_{m}^{f}$. For all $t>0, \psi \in \mathscr{B}$, we have

$$
m\left(F T_{t} \psi\right)=P_{m}^{f} F\left(x_{0}\right) \psi\left(x_{t}\right) f^{t}\left(x_{t}\right)^{-1}=P_{m}^{f}\left\{Y \psi\left(x_{t}\right) f^{t}\left(x_{t}\right)^{-1}\right\}
$$

Since $Y 1_{\alpha<t} \in \mathcal{F}_{<t}$ and $\theta_{t} Y=Y$ a.s. $P_{m}^{f}$, it follows from 5.5.A and 5.4.B that the right side of (0.25) does not depend on $t$. Hence $F \in \mathscr{B}_{m}^{T}$.

Now, by 5.4.B, $P_{m}^{f} Y \psi\left(x_{0}\right) Z=P_{m}^{f} F\left(x_{0}\right) \psi\left(x_{0}\right) Z$ for all $\psi \in \mathscr{B}, Z \in \mathscr{F}_{>0}$. Hence $P_{m}^{f}\left\{Y \mid \mathscr{F}_{>0}\right\}=F\left(x_{0}\right)$ a.s. $P_{m}^{f}$ and, by $(0.24)$

$$
P_{m}^{f}\left\{Y \mid \mathscr{F}_{>t}\right\}=P_{m}^{f}\left\{F\left(x_{0}\right) \mid \mathscr{F}_{\geqslant t}\right\}=F\left(x_{t}\right) \text { a.s. } P_{m}^{f} \text {. }
$$

Letting $t \rightarrow-\infty$, we see that $Y=P_{m}^{f}\left\{Y \mid \mathscr{F}_{\alpha}\right\}=Y_{F}$ a.s. $P_{m}^{f}$.

0.8. Suppose that $\varphi$ is given by formula (0.13). It follows from (0.21) and 5.4.B that

$$
P_{m}^{f} Y n^{\omega}(\varphi)=P_{m}^{f} Y 1_{\alpha<t<\beta} Z
$$

for $Y=Y_{F}$. By Lemma 0.2, (0.26) holds for all $Y \in \mathscr{F}_{\alpha}^{9}$. Formula (0.26) coincides with (0.14) and we establish (0.8) and 0.4.B in the same way as in Subsection 0.6.

\section{REFERENCES}

1. J. L. Doob, Stochastic processes, Wiley, New York, 1953.

2. __ Discrete potential theory and boundaries, J. Math. and Mech. 8 (1959), 433-458.

3. E. B. Dynkin, Exit space of a Markov process, Uspehi Mat. Nauk 24 (148) (1969), 89-152. (English translation: Russian Math. Surveys 24 (1969), 89-157.)

4. __ Excessive measures and entrance laws for a Markov process, Mat. Sb. 84 (126) (1971), 218-253. (English translation: Math. USSR-Sb. 13 (1971), 203-246.)

5. __ Initial and final behaviour of trajectories of Markov processes, Uspehi Mat. Nauk 26 (160) (1971), 153-172. (English translation: Russian Math. Surveys 26 (1971), 165-185.)

6. Integral representation of excessive measures and excessive functions, Uspehi Mat. Nauk 27 (163) (1972), 43-80. (English translation: Russian Math. Surveys 27 (1972), 43-84.) 
7. _ـ Sufficient statistics and extreme points, Ann. Probability 6 (1978), 705-730.

8. On duality for Markov processes, Stochastic Analysis, A. Friedman and M. Pinsky, (eds.), Academic Press, New York, 1978, pp. 63-77.

9. G. A. Hunt, Markov chains and Martin boundaries, Illinois J. Math. 4 (1960), 313-340.

10. _, Transformation of Markov processes, Proc. Internat. Congress Math., Stockholm, 1962, pp. 531-535.

11. H. Kunita and T. Watanabe, Markov processes and Martin boundaries, Part 1, Illinois J. Math. 9 (1965), 485-526.

12. S. E. Kuznecov, Construction of Markov processes with random birth and death times, Teor. Verojatnost. i Primenen. 18 (1973), 596-601. (English translation: Theor. Probability Appl.)

13. R. S. Martin, Minimal positive harmonic functions, Trans. Amer. Math. Soc. 49 (1941), 137-172.

14. J. Neveu, Bases mathématiques du calcul des probabilités, Masson, Paris, 1964.

Department of Mathematics, Cornell University, Ithaca, New York 14853 